\title{
Time-Domain Stochastic Finite Element Simulation of Uncertain Seismic Wave Propagation through Uncertain Heterogeneous Solids
}

\author{
Fangbo Wang ${ }^{\mathrm{a}}$, Kallol Sett ${ }^{\mathrm{a}, *}$ \\ ${ }^{a}$ Department of Civil, Structural and Environmental Engineering, University at Buffalo, \\ The State University of New York, Buffalo, NY, USA
}

\begin{abstract}
This paper presents an efficient numerical methodology in probabilistically solving the governing partial differential equation of solid mechanics with uncertainties in both the material parameter and forcing function in the time domain using the stochastic Galerkin approach. The methodology hypothesizes the input forcing function and the elastic modulus of the solid to be a nonstationary random process and a heterogeneous random field, respectively, and efficiently represents them in terms of multidimensional Hermite polynomial chaos - orthogonal and uncorrelated polynomials of zero-mean, unit variance Gaussian random variables - by taking advantage of the optimality of the Kosambi-Karhunen-Loève theorem. The methodology allows for any non-Gaussian marginal distributions and any arbitrary correlation structures for the input process and field. The solution random processes (displacement, velocity, and acceleration) are also represented in

*corresponding author

Email addresses: fangbowa@buffalo.edu (Fangbo Wang), kallolse@buffalo.edu (Kallol Sett)
\end{abstract}

Preprint submitted to Soil Dynamics and Earthquake Engineering

July 8, 2016

(C) 2016. This manuscript version is made available under the Elsevier user license http://www.elsevier.com/open-access/userlicense/1.0/ 
terms of multidimensional Hermite polynomial chaos expansions whose coefficients at each time step are estimated by applying a stochastic Galerkin projection with the time integration performed via the Newmark's method. The methodology is illustrated, keeping the geotechnical site response analysis in mind, with fully probabilistic, time-domain propagations of bedrock motions through an elastic soil deposit in one-dimension, and is verified using the Monte Carlo method. The effects of input uncertainty parameters of the soil modulus and bedrock motion on the simulated surface motion are also quantified through a parametric sensitivity study.

Keywords: random process, random field, non-Gaussian, non-stationary, polynomial chaos, stochastic finite elements, seismic wave propagation, time domain simulation

\section{Introduction}

Presence of inevitable uncertainties and the need to account for them explicitly in our predictions have long been recognized by the earthquake engineering community. Pioneering works by late Professor C. Allin Cornell during late 1960s [11] and push by the Pacific Earthquake Engineering Research Center (PEER) during early 2000s [12, 47] saw the development of performance-based design framework to account for those uncertainties in our design philosophy. However, numerical simulations of the behavior of solids and structures under seismic loading - which are increasingly being used to feed the performance-based design framework - still remain largely 
deterministic, amid the presence of huge uncertainties in the systems. This is mainly due to the issue of computational tractability of the Monte Carlo approach [46] in solving the governing partial differential equation (PDE) of solid mechanics with uncertain operators/coefficients and uncertain forcing function.

Among alternate approaches for solving uncertain PDEs, there exists analytical technique where the only uncertain parameters are in the external forcing functions. For such a PDE, the probability density function (PDF) of the solution variable satisfies a Fokker-Planck-Kolmogorov (FPK) equation $[33,56]$. Mathematical tools, however, are not that well developed for PDEs with operators/coefficients uncertainty. Exact solution to problems with stochastic operators was attempted by Hopf [28], using the characteristic functional approach. Later, Lee [38] applied the methodology to the problem of wave propagation in random medium and derived an FPK equation, satisfied by the characteristic functional of the random wave field. This characteristic functional approach, though elegant, is not completely rigorous and very difficult to extend in solving realistic problems with irregular geometries and boundaries. The difficulty in analytical solution led to development of approximate techniques. Among the early approximate approaches, the perturbation technique [9] is the most common. It is, however, limited to systems with small uncertainties in model parameters. It also suffers from the closure requirement - higher order statistical moments are needed to compute the lower order statistical moments. Frisch [19] provided a very thorough mathematical review of the early analytical and approximate methods in relation to the theory of (mostly acoustic and electromagnetic) 
wave propagation in random media. Early works related to seismic wave propagation through random geologic media also have mostly relied on the perturbation technique $[59,42,52,64]$. A review of early developments in the field of stochastic soil dynamics was compiled by Manolis [41].

In recent years, with the availability of faster computers, large scale probabilistic simulations are being attempted in other fields of science and engineering using advanced numerical approaches. Among these approaches, the stochastic collocation and the stochastic Galerkin approaches are more common. Stochastic collocation approaches $[4,43,61,63,7]$ can be viewed as a Monte Carlo type sampling technique, with the exception that, instead of at random, the sampling points are selected following some kind of numerical quadrature schemes which are used to estimate the statistical moments of the solution variable. These approaches are non-intrusive approaches as they do not require any modifications to the underlying deterministic code; the stochastic collocation scheme just acts as a wrapper on the deterministic code. Stochastic Galerkin approaches [14, 24, 44, 60, 62, 7], on the other hand, are, in general, intrusive approaches in the sense that they require modifications to the deterministic code; see Refs. [1, 27] regarding non-intrusive stochastic Galerkin approaches. Intrusive stochastic Galerkin approaches usually represent the unknown solution variable using some type of finite series expansions (mostly spectral, e.g., polynomial chaos (PC) expansion [58]) and then employ a Galerkin technique to minimize the errors of finite representation which result in a system of coupled equations. While there is no unanimous agreement in the research community on which approach is better for solving a given problem, it is generally accepted that stochas- 
tic Galerkin approaches are more efficient as their accuracies are optimal $[60,16,6,27]$. In this context, it is important to mention that, depending upon the size of the problem, the stochastic Galerkin method may require inversion of an extremely large matrix. However, the matrix is usually very sparse and has special block properties which are typically exploited for a faster solution $[22,26]$. Moreover, some studies [40, 45] also have demonstrated that solution of such large coupled system of equations may also be advantageous over independently solving many smaller systems of equations (as in the case of the stochastic collocation method) since information on convergence behavior from one block to another can be transmitted during the solution process, resulting in speed up of computation.

In the field of solid mechanics, stochastic Galerkin approaches are so far employed mainly to solve static problems - both linear (elastic) and nonlinear (elastic-plastic as well as geometric) - with uncertain material parameters $[21,23,2,26,55,3,31]$. Solutions of dynamic problems are also attempted, but mostly in the frequency domain [24,25], thereby restricting the use of the algorithms only to linear problems. Kundu and Adhikari [36], very recently, have presented a time domain formulation of a stochastic Galerkin scheme for application in the field of structural dynamics. They, however, have considered uncertainty only in the material parameter - hypothesizing it to be a Gaussian random field while assuming the forcing to be a deterministic function.

We present a time-domain stochastic finite element formulation, based on an intrusive stochastic Galerkin approach, for solving the governing PDE of solid mechanics with uncertainty in both material parameter(s) and forcing 
function. Moreover, our formulation allows for any arbitrary non-Gaussian marginal distributions and any arbitrary heterogeneous/nonstationary correlation structures for the material parameter random field and forcing random process. Conventional approaches for discretizations of input random fields and processes within a stochastic Galerkin scheme typically rely on Kosambi-Karhunen-Loève (KKL) expansion [34, 32, 39] to represent the input random field/process into an optimal number of orthogonal random variables. For Gaussian random fields/processes, the resulting random variables are also uncorrelated (independent), which is a very desirable property as it significantly reduces the overall computational effort. For non-Gaussian random fields/processes, however, the KKL expansion yields correlated random variables which need special treatment, resulting in additional computational burden. Our formulation uses a combination of PC and KKL expansions [54] to efficiently discretize any arbitrary non-Gaussian input random fields/processes directly into a finite number of orthogonal and independent random variables, thereby simplifying the solution process.

The salient features of the formulation are highlighted through two example simulations which are designed keeping the geotechnical site response analysis in mind. Both examples involve one-dimensional propagation of bedrock motion (shear wave) through an elastic soil deposit. While the first example assumes the only uncertainty to be in the soil parameter (shear modulus), the second example considers uncertainties in both the soil parameter and bedrock motion. The simulation results are presented in terms of the marginal mean, marginal standard deviation, and marginal PDF of the surface motion time histories. A parametric study is also performed to 
quantify the effect of the input uncertainty parameters of the soil modulus and bedrock motion on the simulated surface motion.

\section{Formulation of Dynamic Time-Domain Stochastic Finite Ele- ment Method}

The governing equations for a three-dimensional elastic solid in the Cartesian coordinate system are:

$$
\begin{array}{ll}
\frac{\partial \sigma_{i j}}{\partial x_{j}}+b_{i}=\rho \ddot{u}_{i} & \text { (equilibrium equation) } \\
\epsilon_{i j}=\frac{1}{2}\left(\frac{\partial u_{i}}{\partial x_{j}}+\frac{\partial u_{j}}{\partial x_{i}}\right) & \text { (compatibility equation) } \\
\sigma_{i j}=D_{i j k l} \epsilon_{k l} & \text { (constitutive equation) }
\end{array}
$$

where $\sigma, \epsilon, D, \rho, b, u$, and $\ddot{u}$ are the stress, strain, material constitutive parameter, material density, body force, displacement, and acceleration, respectively. Neglecting the body force and employing the Galerkin weak for-

mulation of deterministic, linear, dynamic finite elements [30], the above equations can be written as:

$$
\begin{aligned}
\sum_{e} & {\left[\int_{D_{e}} N_{m}(x) \rho(x) N_{n}(x) d \Omega \ddot{u}_{n}(t)+\right.} \\
& \left.\int_{D_{e}} \nabla N_{m}(x) D(x) \nabla N_{n}(x) d \Omega u_{n}(t)-f_{m}(t)\right]=0
\end{aligned}
$$

where $N_{m}$ is the finite element shape function, while $\sum_{e}$ denotes the assembly procedure over all finite elements of the discretized domain, $\Omega$ and $f_{m}(t)$ incorporates the various elemental contributions to the global force vector. 
We will next assume the material constitutive parameter, $D(x)$, and the forcing function, $f_{m}(t)$, to be a heterogeneous random field and a nonstationary random process, respectively and represent them in terms of multidimensional, Hermite PC expansions with known coefficients. As a result, the nodal displacement, $u_{n}(t)$, and nodal acceleration, $\ddot{u}_{n}(t)$, will also become random processes. They will also be represented using multidimensional, Hermite PC expansions but with unknown coefficients which will be computed using a stochastic Galerkin approach.

\subsection{PC Representations of Material Constitutive Parameter Random Field and Forcing Random Process}

The heterogeneous random field, $D(x)$, may be represented using a multidimensional Hermite PC expansion of dimension $M_{1}$ and order $p$ as [54]:

$$
D(x, \theta)=\sum_{i=0}^{P 1} a_{i}(x) \Psi_{i}\left(\left\{\xi_{r}(\theta)\right\}\right)
$$

where $P_{1}=1+\sum_{s=1}^{p} \frac{1}{s !} \prod_{r=0}^{s-1}\left(M_{1}+r\right)$ and $\left\{\Psi_{i}\right\}$ are multidimensional, orthogonal and uncorrelated, Hermite polynomials of zero-mean, unit variance Gaussian random variables, $\left\{\xi_{r}\right\}$. Note that $\theta$ is introduced to denote uncertainty. The spatially varying coefficients, $\left\{a_{i}\right\}$, of the PC expansion can be computed from the marginal distributions of the constituent random variables (of the target random field) and their correlation behaviors. To this end, following Sakamoto and Ghanem [54], we begin with representing the target random field, $D(x, \theta)$, in a mean-square convergent series:

$$
D(x, \theta)=\sum_{i=0}^{p} \alpha_{i}(x) \Gamma_{i}(\gamma(x, \theta))
$$


where $\left\{\Gamma_{i}\right\}$ are one-dimensional orthogonal polynomials of order $p$ of an underlying zero mean, unit variance Gaussian random field, $\gamma(x, \theta)$. The coefficients $\left\{\alpha_{i}\right\}$ of the series may be computed for each $x$, given the marginal distribution, as:

$$
\alpha_{i}=\frac{\left\langle D \Gamma_{i}\right\rangle}{\left\langle\Gamma_{i}^{2}\right\rangle}
$$

where $\langle\cdot\rangle$ denotes ensemble average operation and $\left\{\left\langle\Gamma_{i}^{2}\right\rangle\right\}$ are the variances of the one dimensional orthogonal Hermite polynomials which may be precomputed symbolically. The numerator on the r.h.s. of Eq. (7) may be evaluated using the inverse transform method with the (ensemble average) integration performed either symbolically or numerically.

Note that the representation of the target random field using Eq. (6) will capture the marginal distributions of the field at every $x$, but not its correlation structure. Hence, the correlation structure of the target random field is next imposed on the representation shown in Eq. (6). This is achieved by expressing the covariance kernel, $C_{\gamma}\left(x_{1}, x_{2}\right)$, of the underlying zero mean, unit variance Gaussian random field, $\gamma(x, \theta)$, in terms of the covariance kernel, $C_{D}\left(x_{1}, x_{2}\right)$, of the target random field, $D(x, \theta)$, through the inverse solution of the following equation with appropriate constraints (see [54]):

$$
C_{D}\left(x_{1}, x_{2}\right)=\sum_{i=1}^{p} \alpha_{i}\left(x_{1}\right) \alpha_{i}\left(x_{2}\right) i ! C_{\gamma}\left(x_{1}, x_{2}\right)^{i}
$$

Kosambi-Karhunen-Loève expansion is then employed to efficiently discretize the zero mean, unit variance Gaussian random field, $\gamma(x, \theta)$, in terms of mutually orthogonal, uncorrelated, zero mean, unit variance Gaussian random variables, $\left\{\xi_{i}\right\}$ : 


$$
\gamma(x, \theta)=\sum_{i=1}^{M_{1}} \sqrt{\lambda_{i}} y_{i}(x) \xi_{i}(\theta)
$$

where $M_{1}$ is the Kosambi-Karhunen-Loève dimension and $\lambda_{i}$ and $y_{i}$ are the eigenvalue and eigenvector, respectively, of the covariance kernel, $C_{\gamma}\left(x_{1}, x_{2}\right)$, of the zero mean, unit variance Gaussian random field, $\gamma(x, \theta)$. The eigenvalues and eigenvectors are the solution of a Fredholm's integral equation of the second kind:

$$
\int_{\Omega_{D}} C_{\gamma}\left(x_{1}, x_{2}\right) y_{n}\left(x_{1}\right) d x_{1}=\lambda_{n} y_{n}\left(x_{2}\right)
$$

with the following unit variance constraint:

$$
\sum_{i}\left(\sqrt{\lambda_{i}} y_{i}(x)\right)^{2}=1
$$

Eq. (10) may be solved using, among others, the finite element method [24], which necessitates approximation of each eigenfunction, $y_{n}$, using the standard shape function, $h_{i}(x)$, as:

$$
f_{n}(x)=\sum_{i=1}^{N_{K L}} y_{i n} h_{i}(x)
$$

where $N_{K L}$ is the number of finite element nodes and $y_{\text {in }}$ is the nodal value of the eigenfunction, $y_{n}$. Utilizing the above representation and requiring the error to be orthogonal to the approximating space, Eq. (10) may be written in the following weak form,

$$
\sum_{i=1}^{N_{K L}} y_{i n}\left[\int_{\Omega_{D}} \int_{\Omega_{D}} C_{\gamma}\left(x_{1}, x_{2}\right) h_{i}\left(x_{2}\right) h_{j}\left(x_{1}\right) d x_{1} d x_{2}-\lambda_{n} \int_{\Omega_{D}} h_{i}(x) h_{j}(x) d x\right]=0
$$


which represents a generalized eigenvalue problem and can be solved for $\lambda_{n}$ and $y_{i n}$. The number of finite element nodes, $N_{K L}$, needed for accuracy depends upon the correlation structure of the random field [29]. A fluctuating correlation structure typically requires a finer mesh and vice versa. If $N_{K L}$ number of eigenvalues and eigenvectors are obtained from a finite element analysis, but only $M_{1}$ number of eigenvalues and eigenvectors are used to represent the Gaussian random field, $\gamma(x, \theta)$, using the Kosambi-KarhunenLoève theorem, then Eq. (9) must be normalized to satisfy the unit variance constraint (Eq. (11)) as:

$$
\gamma(x, \theta)=\sum_{i=1}^{M_{1}} \frac{\sqrt{\lambda_{i}} y_{i}(x)}{\sqrt{\sum_{m=1}^{M_{1}}\left\{\sqrt{\lambda_{m}} y_{m}(x)\right\}^{2}}} \xi_{i}(\theta)
$$

Finally, equating the two representations of $D(x, \theta)$ in Eqs. (5) and (6) we can find the coefficients, $\left\{a_{i}\right\}$, of Eq. (5) as [54]:

$$
a_{i}(x)=\frac{p !}{\left\langle\Psi_{i}^{2}\right\rangle} \alpha_{p}(x) \prod_{j=1}^{p} \frac{\sqrt{\lambda_{r_{(j)}}} y_{r_{(j)}}(x)}{\sqrt{\sum_{m=1}^{M_{1}}\left\{\sqrt{\lambda_{m}} y_{m}(x)\right\}^{2}}}
$$

Similarly, the nonstationary forcing random process, $f_{m}(t)$, may be represented using another multidimensional Hermite PC expansion of dimension $M_{2}$ and order $q$ as:

$$
f_{m}(t, \theta)=\sum_{j=0}^{P_{2}} f_{m j}(t) \Psi_{j}\left(\left\{\xi_{k}(\theta)\right\}\right)
$$

where $P_{2}=1+\sum_{s=1}^{q} \frac{1}{s !} \prod_{r=0}^{s-1}\left(M_{2}+r\right)$ and the time varying coefficients, $\left\{f_{m j}\right\}$ can be computed as: 


$$
f_{m j}(t)=\frac{q !}{\left\langle\Psi_{j}^{2}\right\rangle} \phi_{m q}(t) \prod_{l=1}^{q} \frac{\sqrt{v_{k_{(l)}}} z_{k_{(l)}}(t)}{\sqrt{\sum_{n=1}^{M_{2}}\left\{\sqrt{v_{n}} z_{n}(t)\right\}^{2}}}
$$

In Eq. (17) the coefficient, $\phi$, can be calculated, after dividing the domain of the random process in a uniform grid, from the marginal distribution at each grid point, while the eigenvalue, $v$, and eigenvector, $z$, can be obtained through the Kosambi-Karhunen-Loève eigenanalysis on the covariance kernel of the random process.

Next, we will represent the response random processes, $u_{n}(t)$ and $\ddot{u}_{n}(t)$, also in terms of multidimensional Hermite PC expansions but with unknown coefficients which will be computed through a stochastic Galerkin approach.

\subsection{Stochastic Galerkin Approach to Compute the PC Coefficients of Dis- placement and Acceleration Random Processes}

The dimension of a multidimensional PC expansion denotes the number of independent random variables that are used to synthesize the target random field or process. So, if $M_{1}$ independent random variables are used to represent the material constitutive parameter, $D(x, \theta)$, and another $M_{2}$ independent random variables are used to represent the the forcing function, $f_{n}(t, \theta)$, then it is postulated that the response processes, $u_{n}(t)$ and $\ddot{u}_{n}(t)$, will be functions of $M_{1}+M_{2}$ independent random variables. Accordingly, let's represent the nodal displacement, $u_{n}(t)$, in terms of a multidimensional Hermite PC expansion of dimension $M_{1}+M_{2}$ and order $Q$ as:

$$
u_{n}(t, \theta)=\sum_{k=0}^{P_{3}} d_{n k}(t) \Psi_{k}\left(\left\{\xi_{l}(\theta)\right\}\right)
$$


where $P_{3}=1+\sum_{s=1}^{Q} \frac{1}{s !} \prod_{r=0}^{s-1}\left(M_{1}+M_{2}+r\right)$. Twice differentiating Eq. (18), we obtain a multidimensional Hermite PC representation of nodal acceleration, $\ddot{u}_{n}(t)$, as:

$$
\ddot{u}_{n}(t, \theta)=\sum_{k=0}^{P_{3}} \ddot{d}_{n k}(t) \Psi_{k}\left(\left\{\xi_{l}(\theta)\right\}\right)
$$

Substituting Eqs. (5), (16), (18), and (19) into Eq. (4), and denoting the shape function gradients as:

$$
\nabla N_{n}(x):=B(x)
$$

we obtain:

$$
\begin{aligned}
\sum_{e} & {\left[\sum_{k=0}^{P_{3}} \int_{D_{e}} N_{m}(x) \rho(x) N_{n}(x) d \Omega \Psi_{k} \ddot{d}_{n k}(t)+\right.} \\
& \left.\sum_{k=0}^{P_{3}} \sum_{i=0}^{P_{1}} \int_{D_{e}} B_{m}(x) a_{i}(x) B_{n}(x) d \Omega \Psi_{i} \Psi_{k} d_{n k}(t)-\sum_{j=0}^{P_{2}} f_{m j}(t) \Psi_{j}\right]=0
\end{aligned}
$$

Multiplying both sides of Eq. (21) by $\Psi_{l}$ and taking ensemble average, we obtain the following system of ordinary differential equations:

$$
\begin{aligned}
& \sum_{n=1}^{N} \sum_{k=0}^{P_{3}}\left\langle\Psi_{k} \Psi_{l}\right\rangle \int_{D_{e}} N_{m}(x) \rho(x) N_{n}(x) d \Omega \ddot{d}_{n k}(t)+ \\
& \quad \sum_{n=1}^{N} \sum_{k=0}^{P_{3}} \sum_{i=0}^{P_{1}}\left\langle\Psi_{i} \Psi_{k} \Psi_{l}\right\rangle \int_{D_{e}} B_{m}(x) a_{i}(x) B_{n}(x) d \Omega \quad d_{n k}(t)=\sum_{j=0}^{P_{2}}\left\langle\Psi_{j} \Psi_{l}\right\rangle f_{m j}(t)
\end{aligned}
$$


with $l=0,1,2, \ldots, P_{3}$ and $m=1,2, \ldots, N$ where $N$ is the number of finite element nodes. The form of Eq. (22) is very similar to that of the deterministic finite element system of equations with the exception of the additional "stochastic" degrees of freedom arising out of the non-zeroth PC terms. Note that when $P_{1}=P_{2}=P_{3}=0$, Eq. (22) becomes identical to the deterministic finite element system of equations. Writing Eq. (22) in the familiar matrix-vector form results in:

$$
M \ddot{d}+K d=F
$$

where $M$ and $K$ may be termed as the generalized mass and stiffness matrices, while $F, d$, and $\ddot{d}$ may be termed as the generalized force, displacement, and acceleration vectors, respectively. Note that the ensemble averages of the double and triple products of the PC basis functions appearing within $M, K$, and $F$ may be pre-computed symbolically. Introduction of a mass and stiffness proportional damping matrix transforms Eq. (23) to:

$$
M \ddot{d}+C \dot{d}+K d=F
$$

where $\boldsymbol{C}=\alpha \boldsymbol{M}+\beta \boldsymbol{K}$, with $\alpha$ and $\beta$ being the Rayleigh damping parameters. Eq. (23) or Eq. (24) may be solved using any of the available time integration schemes of the deterministic dynamic finite element method. Note that, depending upon the number of PC terms used to represent the displacement random process, the size of the stochastic finite element system of equations can be substantially larger than the corresponding deterministic finite element system of equations. However, the ensemble averages of the double and triple products of the PC basis functions induce special block sparsities in 
the $M, C$, and $K$ matrices that may be exploited while solving the stochastic finite element system of equations. Further note that even though the above formulation assumes linear elastic material constitutive behavior, it is general enough to be extended to elastic-plastic solids for which only the generalized stiffness matrix, $K$, needs to be updated after each time step following an appropriate elastic-plastic constitutive equation. An elastic-plastic constitutive equation can be probabilistically integrated at each material integration (Gauss) point using the strain from the finite element level by employing, among others, a Fokker-Planck-Kolmogorov equation approach [55] to provide updated generalized (tangent) stiffness matrix for the next finite element time step - the details of which, however, is beyond the scope of this paper.

\subsection{Estimation of Statistics of Displacement and Acceleration Random Pro- cesses from Computed PC Coefficients}

Having obtained the multidimensional PC coefficients of displacement and acceleration random processes, they can be substituted in Eqs. (18) and (19) to synthesize the random processes. Realizations of the processes can then be generated simply through random sampling.

If only the evolutionary statistics up to the second order is sought for, then they can be directly computed from the PC coefficients, utilizing some of the properties of the $\mathrm{PC}$ basis functions. For example, the evolutionary mean and standard deviation of displacement time history at node $n$ may be estimated as:

$$
\mu_{u_{n}}(t)=\left\langle u_{n}(t, \theta)\right\rangle=d_{n 0}(t)
$$

and, 


$$
\sigma_{u_{n}}(t)=\sqrt{\sum_{k=0}^{P_{3}}\left\langle\Psi_{k}^{2}\right\rangle\left(d_{n k}(t)\right)^{2}}
$$

The evolutionary PDFs of displacement and acceleration time histories can also be estimated either through the use of the Edgeworth's series [24] or by simply generating a statistically significant number of realizations of the variable of interest, followed by estimating the distribution at each $t$ using the kernel density estimation technique.

\section{Simulation Results and Discussions}

This section exemplifies the above-presented stochastic finite element framework by probabilistically simulating propagation of bedrock seismic shear wave through a fictitious $10 \mathrm{~m}$ thick elastic soil deposit at a site (latitude: $34.5^{\circ}$; longitude: $-118.2^{\circ}$ ) in Los Angeles. The soil deposit is modeled using 10 one-dimensional shear elements. The soil shear modulus is assumed to be uncertain. It is hypothesized to be a homogeneous lognormal random field with a marginal mean value of $100 \mathrm{MPa}$, a marginal standard deviation of $40 \mathrm{MPa}$, a first-order Markov (exponential) correlation structure, and a correlation length of $5 \mathrm{~m}$; all the above assumed uncertainty parameters are typical for soils $[15,37,50,51,17,18,5]$. Two examples are presented. The first example considers the bedrock motion to be deterministic. To this end, a site-specific response spectrum is first obtained, assuming a hazard level of $10 \%$ probability of exceedance in 50 years, using a combination of

2008 Interactive United States Geological Survey Deaggregation tool [57] and the Caltrans ARS online tool [10]. Then, a past-recorded ground motion is 
scaled, using the PEER online tool [49], to match the above-obtained response spectrum. The second example, on the other hand, considers the bedrock motion to be a non-stationary random process. Using the PEER online tool, two hundred past-recorded ground motions are scaled to match the same site-specific response spectrum. Those motions are assumed to be the realizations of the bedrock random process. The bedrock motion and the soil shear modulus are assumed to be statistically independent. In this context, it is important to mention that the use of spectrally-matched real time histories in characterizing the bedrock motion is just for the illustration of the presented methodology. The formulation, however, is general enough to accommodate any other types of bedrock motion characterization techniques including physics-based (e.g., [13]), pure stochastic (e.g., [53]) and hybrid (e.g., [8]) approaches. The suitability of one technique over another is beyond the scope of this paper. For both the examples, bedrock motions are applied to the soil finite elements using the penalty stiffness approach.

\subsection{Example 1: Shear modulus is uncertain; bedrock motion is deterministic}

The deterministic bedrock motion, which is a scaled 1970 Lytle Creek, California motion recorded at the Devil's Canyon station, is shown in Figure 1. Note that a scale factor of 6.9035 is used to match the site-specific response spectrum.

The uncertain shear modulus, which is assumed to be a homogeneous lognormal random field, is synthesized using the multi-dimensional Hermite PC expansion, Eq. (5) with the coefficients given by Eq. (15). The study by Sakamoto and Ghanem [54] has shown that the PC-synthesized correlation structure and marginal distribution are independent of the order and dimen- 


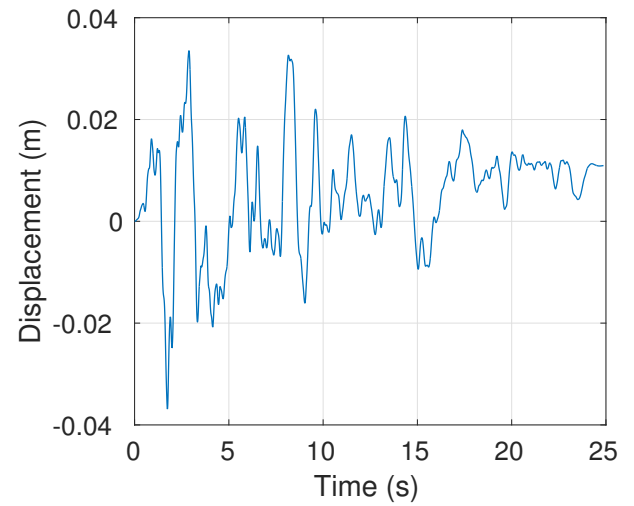

(a)

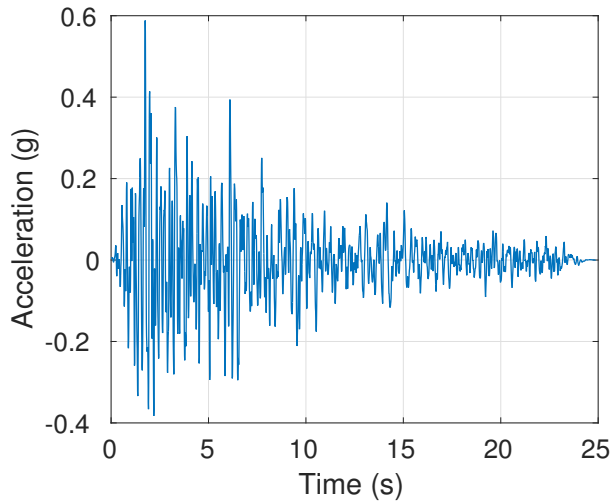

(b)

Figure 1: Deterministic bedrock motion (a scaled 1970 Lytle Creek, California motion): (a) Displacement and (b) acceleration.

sion, respectively. Accordingly, an appropriate dimension for the modulus PC expansion is first selected by varying the dimension while keeping the order of Eq. (5) constant, and comparing the resulting correlation structures with the target correlation structure (first-order Markov with a correlation length of $5 \mathrm{~m}$ ), as shown in Figure 2. Note that the underlying Kosambi-KarhunenLoève eigenanalysis is performed numerically using the finite element method by discretizing the domain of the random field using 100 elements and thus, 101 eigenvalues and eigenvectors are obtained. But only the first 2, 4, 5, and 15 eigenvalues and eigenvectrors are used for computing the PC coefficients. Further note that the synthesized coefficients of correlation plotted in Figure 2 are calculated from the PC coefficients, $\left\{a_{i}\right\}$, as:

$$
\rho_{D}^{\text {syn }}(x)=\frac{\sum_{i=1}^{P_{1}} a_{i}(0) a_{i}(x)\left\langle\Psi_{i}^{2}\right\rangle}{\sqrt{\sum_{j=1}^{P_{1}} a_{j}^{2}(0)\left\langle\Psi_{j}^{2}\right\rangle} \sqrt{\sum_{k=1}^{P_{1}} a_{k}^{2}(x)\left\langle\Psi_{k}^{2}\right\rangle}}
$$


and the target coefficients of correlation are calculated using the following equation:

$$
\rho_{D}^{\text {exact }}(x)=e^{-x / l_{c}}
$$

where $x$ is the lag distance and $l_{c}(=5 \mathrm{~m})$ is the correlation length. As can be observed from Figure 2 that the synthesized correlation structure converges to the target correlation structure as the dimension of the PC expansion is increased and a dimension of 5 may be reasonable for the modulus PC expansion.

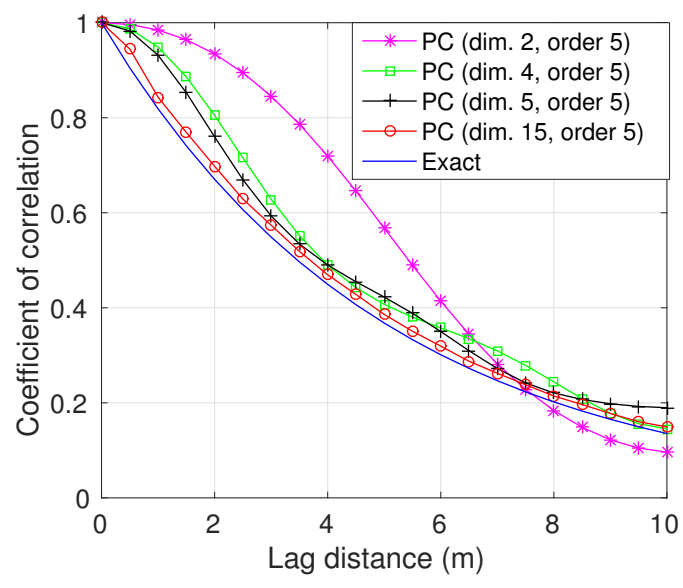

Figure 2: PC-synthesized correlation structure of the shear modulus random field.

Next, an appropriate order for the modulus PC expansion is selected by varying the order while keeping the dimension of Eq. (5) constant, and comparing the resulting marginal PDFs with the target marginal PDF (a lognormal density function with a mean of $100 \mathrm{MPa}$ and a standard deviation of $40 \mathrm{MPa}$ ), as shown in Figure 3. As the marginal distribution doesn't vary in space for a homogeneous field, the PDFs are compared only at one location 
in the domain - at the center of the soil deposit. Note that the synthesized PDFs are estimated by employing the kernel density estimation technique after generating a million realizations of $D$ for each case at the center of the soil deposit $(x=5 \mathrm{~m})$ using Eq. (5). As can be observed from Figure 3 that the synthesized marginal PDF converges to the target marginal PDF as the order of the PC expansion is increased and an order of 2 may be reasonable for the modulus PC expansion.

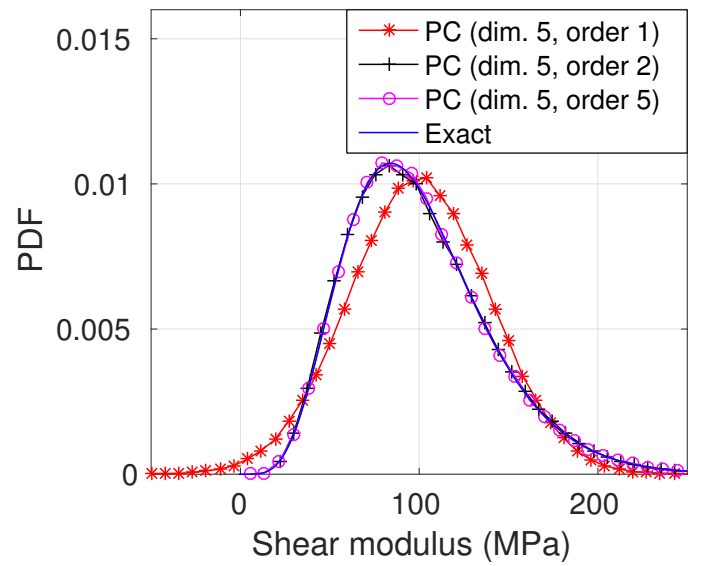

Figure 3: PC-synthesized marginal PDF of the shear modulus random field at the center of the soil deposit.

Having obtained the coefficients of the PC expansion for the shear modulus random field and selected the bedrock motion, Eq. (22) may now be solved to simulate (deterministic) seismic wave propagation through uncertain soils. Note that since the bedrock motion is deterministic, the r.h.s. of Eq. (22) simplifies to $\left\langle\Psi_{0} \Psi_{l}\right\rangle f_{m 0}(t)=\left\langle\Psi_{l}\right\rangle f_{m}(t)$ as $P_{2}=0$, and the dimension of the displacement PC expansion becomes equal to the dimension of the shear modulus PC expansion. This study has introduced a mass and 

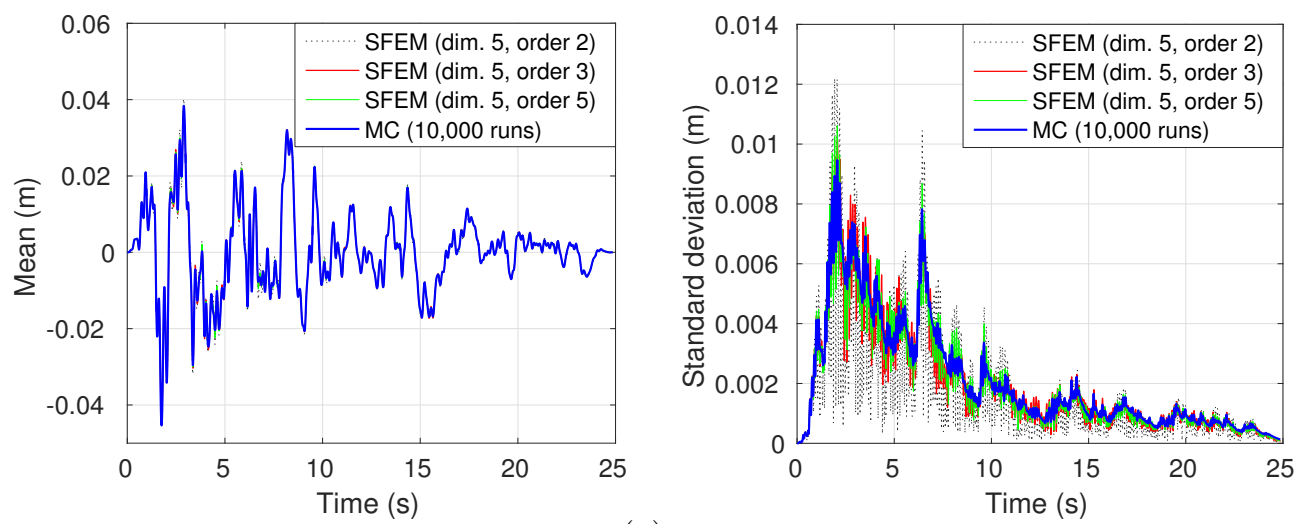

(a)
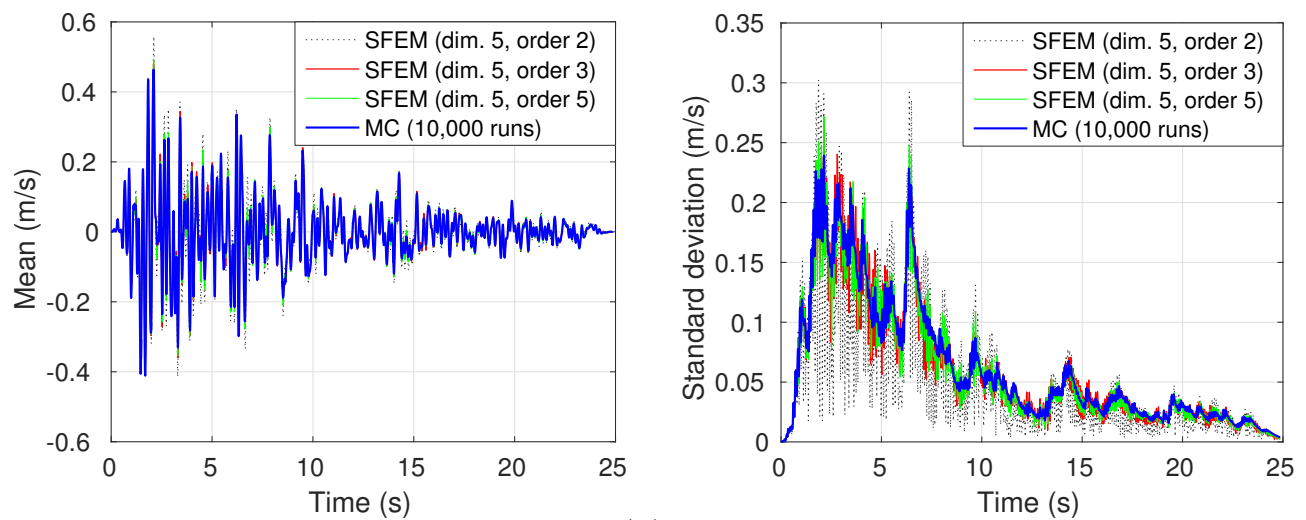

(b)


(c)

Figure 4: Simulated marginal mean and standard deviation of (a) displacement, (b) velocity, and (c) acceleration at the surface when only the shear modulus is uncertain. 
stiffness proportional (Rayleigh) damping to the system and employed the the Newmark's method [48] to solve the governing stochastic finite element system of ordinary differential equations (Eq. (24)). The Rayleigh damping parameters, $\alpha$ and $\beta$, are computed assuming a $5 \%$ critical damping for the first generalized and natural modes of vibration. The first generalized frequency is obtained by solving a generalized eigenvalue problem with the generalized mass and stiffness matrices, while the first natural frequency, $\omega_{1}$ is calculated using the formula [35]: $\omega_{1}=\pi V_{s} / 2 H$, where $H$ is the thickness of the soil deposit and $V_{s}$ is the soil shear wave velocity which can be calculated from the shear modulus, $G$, and density, $\rho$, as $V_{s}=\sqrt{G / \rho}$. The resulting system of algebraic equations is solved, with a time step size of 0.01 second, by employing Matlab's backslash command which uses a built-in algorithm in selecting an appropriate solver depending upon the sparsity and other properties of the input (Newmark's effective stiffness) matrix.

Shown in Figure 4 are the simulated mean and standard deviation of surface displacement, velocity, and acceleration time histories with varying order of displacement PC expansion. A conventional, brute-force Monte Carlo simulation results are also shown. The Monte Carlo simulation is performed by first (1) generating $N$ number of realizations of the shear modulus random field. It is achieved, following [5], by (a) dividing the domain of the random field into a uniform grid of $n$ points, (b) generating an $n \times n$ correlation matrix using the given correlation function, (c) decomposing the correlation matrix into an upper triangular matrix using the Cholesky algorithm, (d) multiplying the transpose of the upper triangular matrix by a vector of $n$ uncorrelated standard Gaussian random numbers to obtain a vector of $n$ 
correlated standard Gaussian random numbers with the desired correlation structure, (e) transforming the vector of $n$ correlated standard Gaussian random numbers into a vector of $n$ correlated lognormal random numbers by using the inverse CDF transformation using the given mean and standard deviation to obtain one realization of the shear modulus random field, and (f) repeating Steps (a)-(e) to obtain $N$ number of realizations. Next, (2) the bedrock motion is deterministically propagated through the soil deposit for each instance of the soil profile to obtain $N$ number of realizations of surface motion time histories. The deterministic wave propagation is simulated by using the same code that is developed for the stochastic finite element simulation but by setting $P_{1}=P_{2}=P_{3}=0$ and applying the damping to the first and second natural modes of vibration. Finally, (3) the $N$ number of realizations of surface motion time histories are statistically post-processed to compute the marginal mean, standard deviation, and PDF of surface displacement, velocity, and acceleration time histories. As can be observed from Figure 4 that the stochastic finite element mean and standard deviation behaviors of displacement, velocity, and acceleration time histories converge to the respective Monte Carlo result as the order of displacement PC expansion is increased and an order higher than that is used for the shear modulus PC expansion is needed to get reasonable matches, especially for the standard deviation time histories, with the Monte Carlo results. This is because of the nature of the marginal distributions of the surface motion time histories. The marginal PDF of the surface acceleration time history at $8 \mathrm{~s}$ is shown in Figure 5. As can be observed that the marginal PDF of the surface acceleration is more non-Gaussian than the marginal PDF of the shear modulus 
random field. Theoretically, the more the deviation of a non-Gaussian PDF from its Gaussian counterpart, the higher the order of Hermite PC expansion that is needed to accurately represent it.

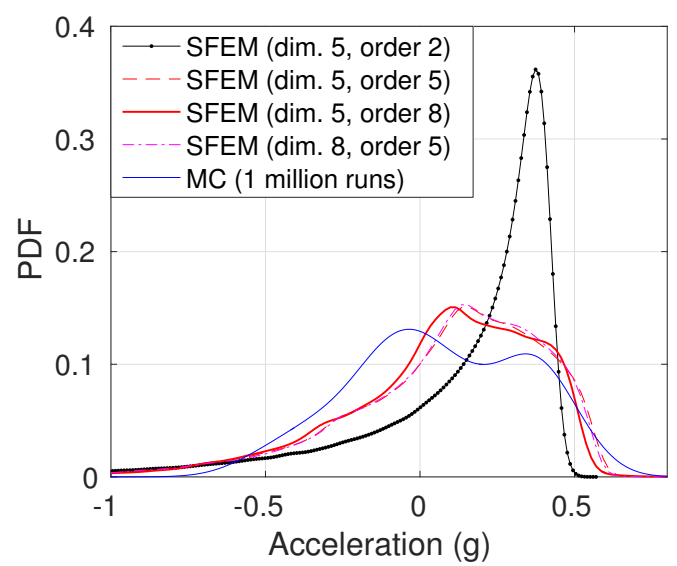

Figure 5: Simulated marginal PDF of surface acceleration at $8 \mathrm{~s}$ when only the shear modulus is uncertain.

The computation time needed to solve Example 1 using the stochastic finite element method with a dimension 5 , order $5 \mathrm{PC}$ representation for the displacement random process - which yields a reasonable accuracy - is 5 minutes, whereas that using a ten thousand and a million runs of the conventional Monte Carlo method are about 35 minutes and 58 hours, respectively. Note that a ten thousand Monte Carlo runs may be sufficient if one is only interested in statistics up to the second order, while a million runs may be needed for accuracy of the evolutionary PDF. Further note that the computation time of the stochastic finite element simulation does not include the time taken to compute the ensemble averages of the double and triple products of the PC basis functions. 


\subsection{Example 2: Both shear modulus and bedrock motion are uncertain}

The penalty stiffness approach of applying the bedrock motion to the soil finite element model multiplies the bedrock displacement by a large (penalty) stiffness to convert it to the nodal force. Accordingly the bedrock displacement, which is a nonstationary random process, is represented using the PC expansion. To this end, the statistics - the correlation matrix and the evolutionary marginal mean, standard deviation and distribution - that are needed to compute the PC coefficients are estimated by analyzing the 200 realizations of the nonstationary random process. The correlation matrix is generated by dividing the domain of the random process in a uniform grid with grid points equispaced at 0.01 second. The marginal mean values, standard deviation values and distributions are also estimated at every 0.01 second. Kolmogorov-Smirnov test on the displacement data at each grid point has showed that a Gaussian assumption for the marginal distributions is acceptable at a 5\% significance level. It implies that a Hermite PC expansion of order 1 should be sufficient for the bedrock random process. Next, an appropriate dimension for the bedrock PC expansion is selected by varying the dimension and comparing the synthesized marginal mean and standard deviation values of the displacement as well as velocity, and acceleration with the their respective target, as shown in Figure 6. Note that the velocity and acceleration PC coefficients are obtained from the displacement PC coefficients through differentiation. Further note that the underlying Kosambi-Karhunen-Loève eigenanalysis is performed on the same grid that is used for generation of the correlation matrix i.e., with a finite element size of 0.01 second. 

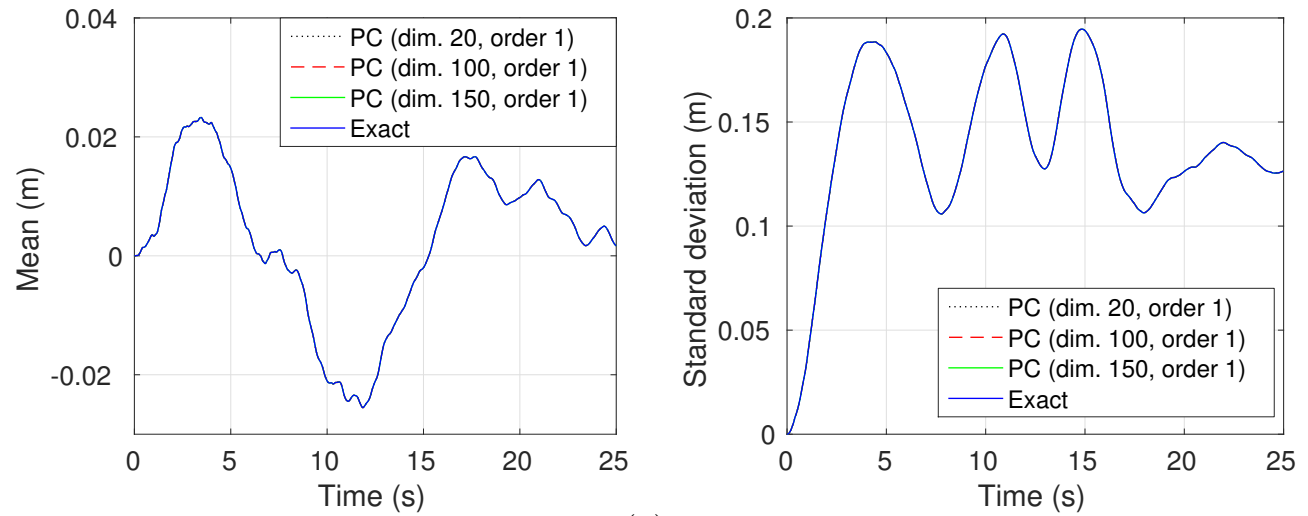

(a)
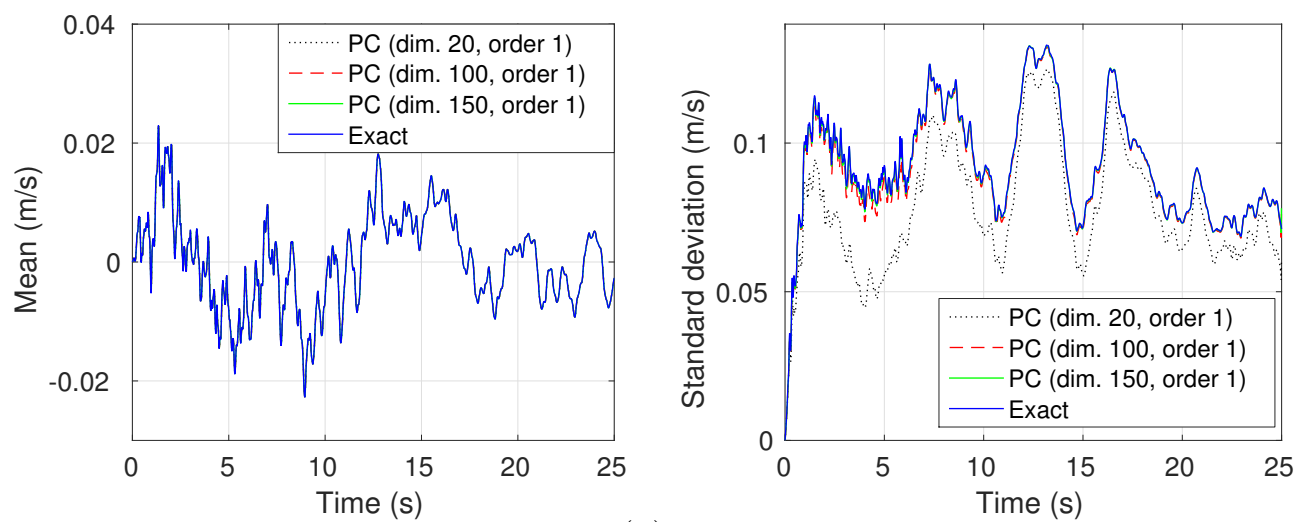

(b)
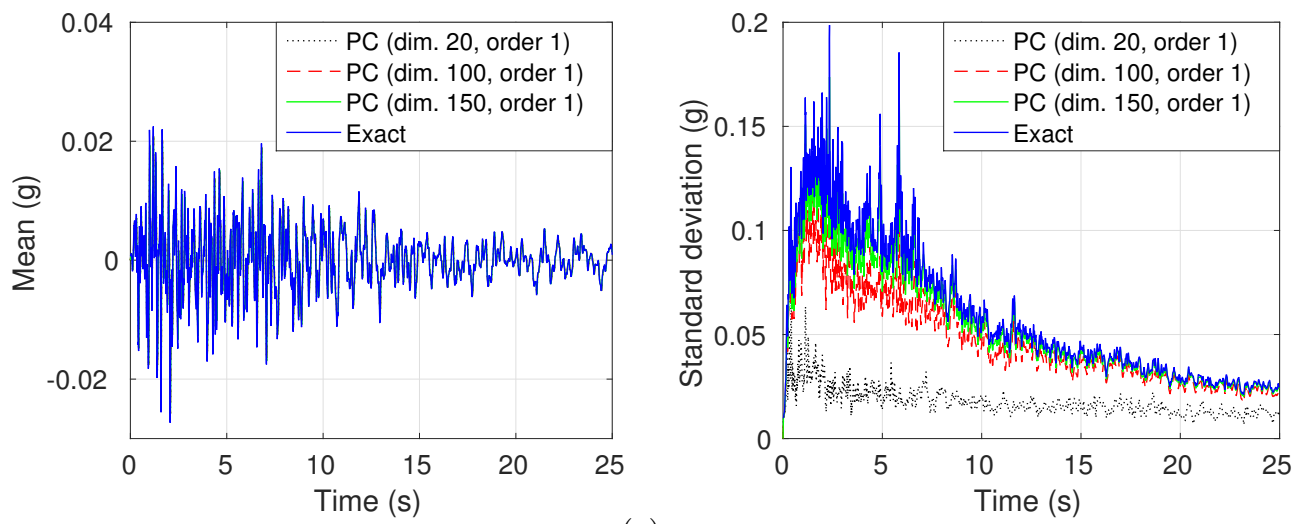

(c)

Figure 6: PC-synthesized marginal mean and standard deviation behaviors of the bedrock motion random process: (a) Displacement, (b) velocity, and (c) acceleration. 
As can be observed from Figure 6 that marginal standard deviation behaviors of displacement as well as velocity and acceleration converge to the respective exact behavior when the dimension of $\mathrm{PC}$ expansion is increased and that even though a relatively lower dimension is sufficient to get a match for the displacement, a higher dimension of the PC expansion is needed for the velocity and acceleration. This is because of the different correlation structures of the bedrock displacement, velocity, and acceleration random processes. The acceleration random process is less correlated in time than the velocity random process which is even less correlated than the displacement random process. Hence, while a fewer number of independent random variables are enough to capture the displacement behavior, a larger number of independent random variables are required to capture the velocity and acceleration behaviors. Figure 7 compares the correlation structures of the bedrock displacement and acceleration random processes synthesized using a PC expansion with a dimension of 150 and an order of 1 - which yields reasonable matches for marginal standard deviation behaviors of both displacement and acceleration - with the respective exact correlation structure. Note that the synthesized coefficients of correlation plotted in Figure 7 are computed from the bedrock displacement/acceleration PC coefficients, $\left\{\kappa_{i}\right\}$, as:

$$
\rho_{\text {bedrock }}^{\text {syn }}\left(t_{1}, t_{2}\right)=\frac{\sum_{i=1}^{P_{2}} \kappa_{i}\left(t_{1}\right) \kappa_{i}\left(t_{2}\right)\left\langle\Psi_{i}^{2}\right\rangle}{\sqrt{\sum_{j=1}^{P_{2}} \kappa_{j}^{2}\left(t_{1}\right)\left\langle\Psi_{j}^{2}\right\rangle} \sqrt{\sum_{k=1}^{P_{2}} \kappa_{k}^{2}\left(t_{2}\right)\left\langle\Psi_{k}^{2}\right\rangle}}
$$

Further note that only the displacement PC coefficients are computed using the approach described in Section 2.1 while the acceleration PC coefficients are obtained by double differentiating the displacement $\mathrm{PC}$ coefficients in 
time. In this context, we would like to mention that while selecting an appropriate dimension for the bedrock motion PC expansion, it is not necessary to compare the synthesized and exact correlation structures. Just comparing the marginal standard deviation behaviors will be sufficient. If the correlation structures are not captured correctly, then the marginal standard deviation behaviors will not match.

Exact

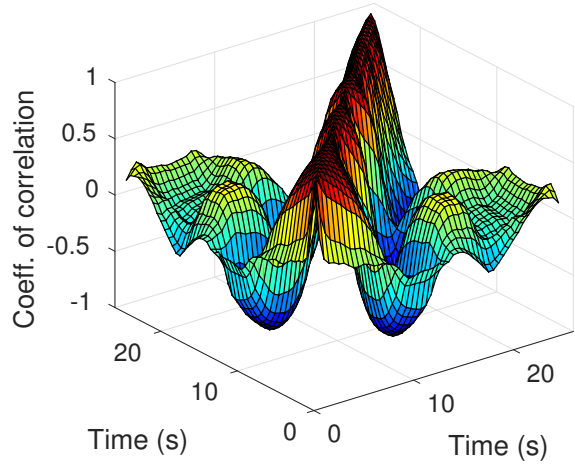

\section{Synthesized}

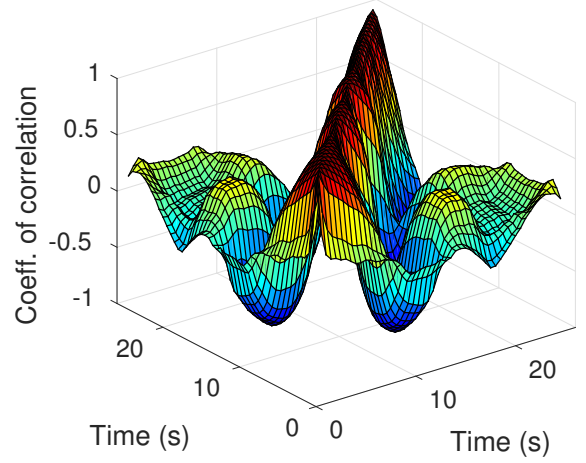

(a)

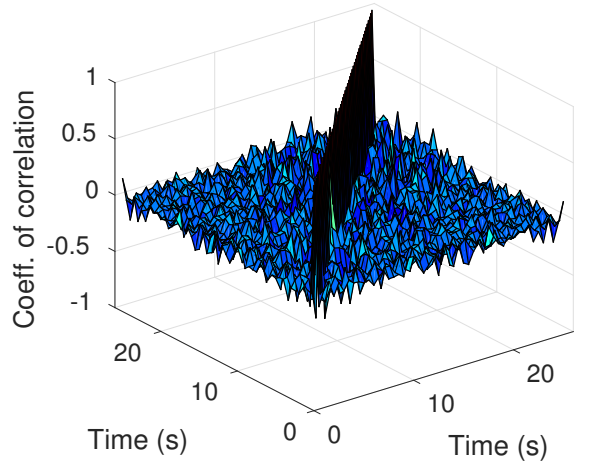

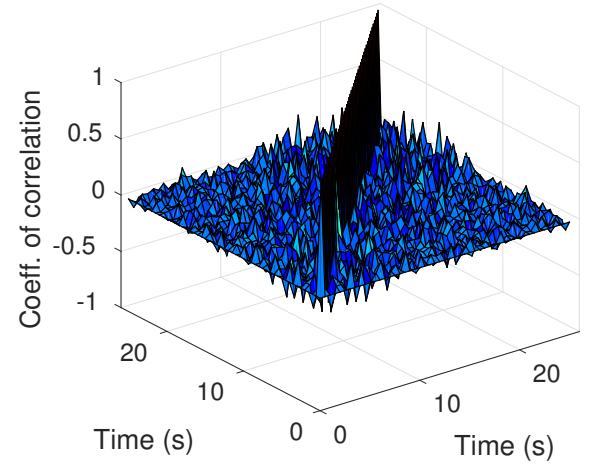

(b)

Figure 7: Correlation structure of the bedrock motion random process synthesized with a PC expansion of dimension 150 and order 1: (a) Displacement and (b) acceleration. 
Figure 8 shows the marginal PDFs of bedrock displacement and acceleration at $8 \mathrm{~s}$ and $15 \mathrm{~s}$, synthesized with a $\mathrm{PC}$ expansion of dimension 150 and order 1 . They compare well with the respective target PDF which corroborates the Kolmogorov-Smirnov test findings that the marginal distributions are indeed Gaussian. A higher-order PC synthesization results are also shown. As expected, the results do not differ much.
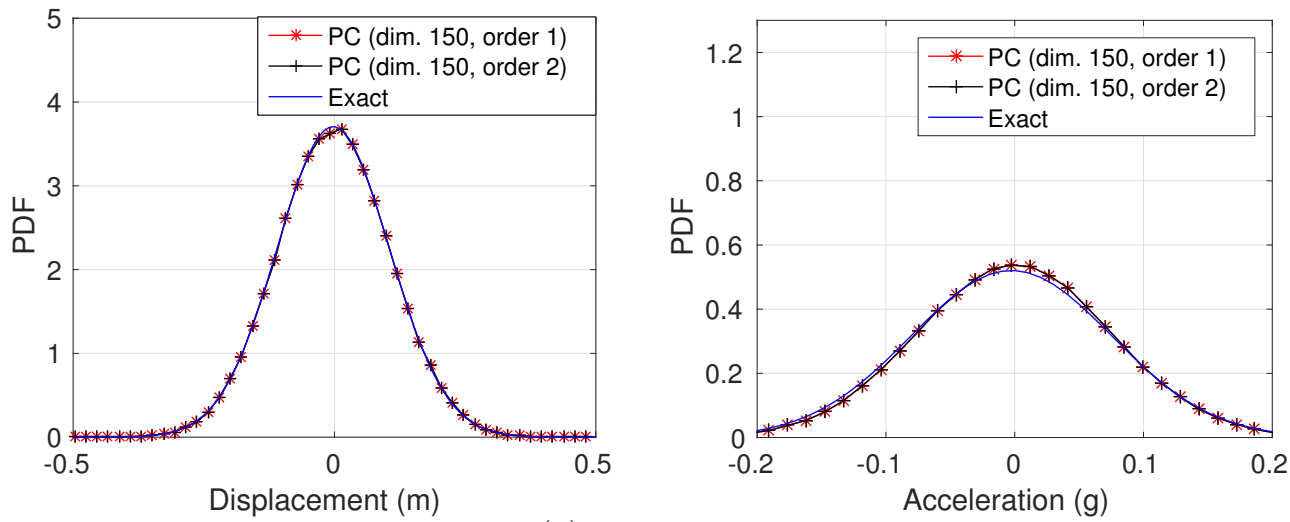

(a) time $=8 \mathrm{~s}$
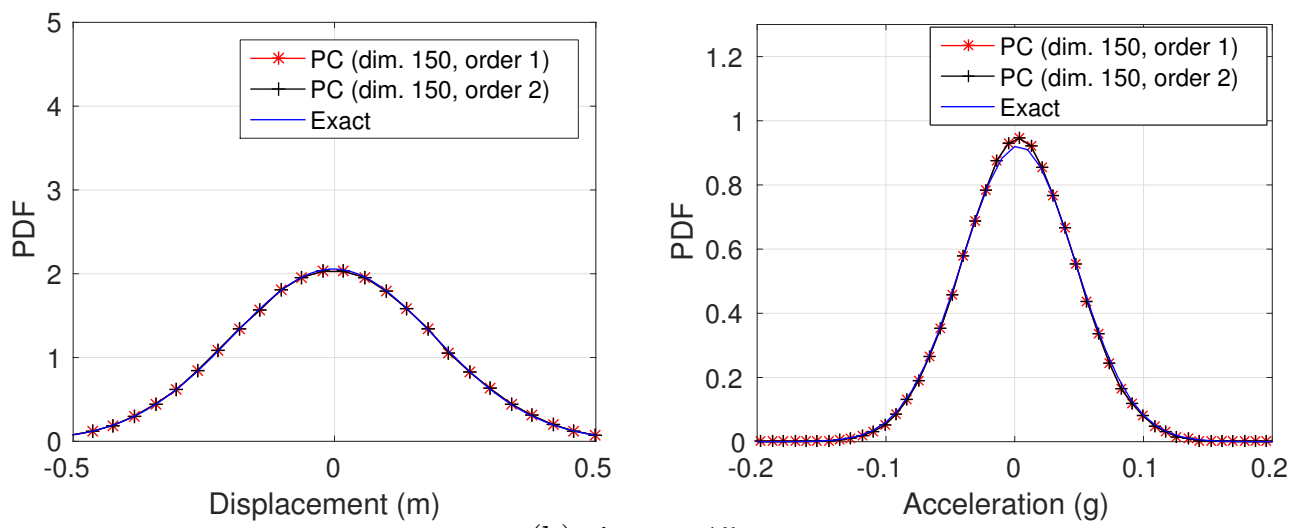

(b) time $=15 \mathrm{~s}$

Figure 8: PC-synthesized marginal PDF of bedrock displacement and acceleration random processes at (a) 8s and (b) 15s. 



(a)
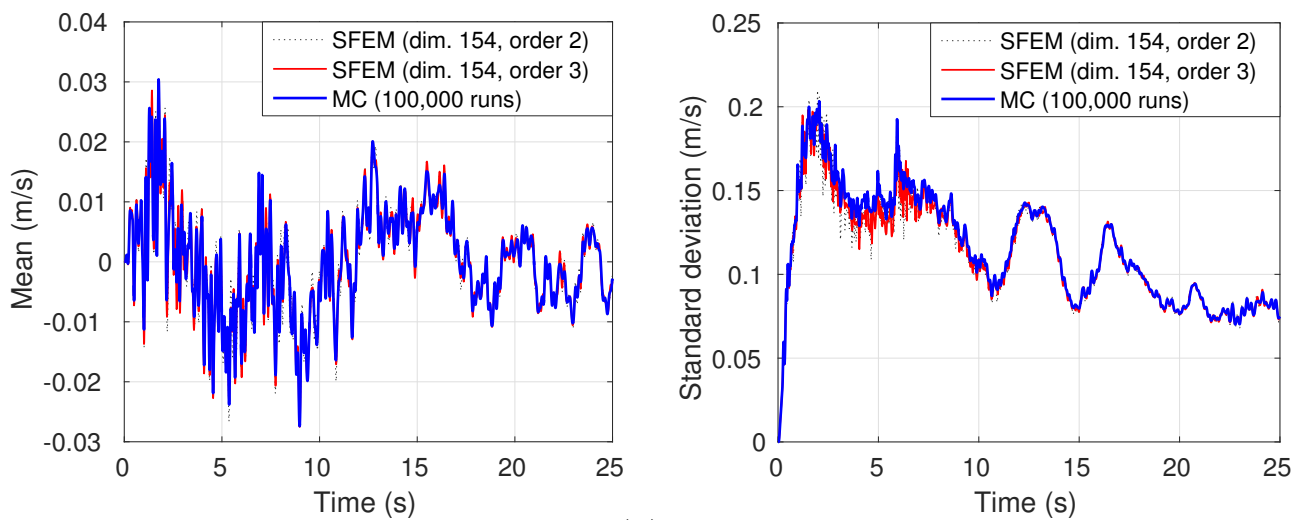

(b)


(c)

Figure 9: Simulated marginal mean and standard deviation of (a) displacement, (b) velocity, and (c) acceleration at the surface when both the shear modulus and bedrock motion are uncertain. 
Having selected the dimension and order of the bedrock motion PC expansion and already obtained the PC coefficients for the shear modulus random field (same as Example 1) from Section 3.1, Eq. (24) is now solved to simulate uncertain seismic wave propagation through uncertain elastic soil deposit. The damping coefficient and the time step size are kept the same as in Example 1. The simulation results are shown in Figure 9 in terms of the marginal mean and standard deviation behaviors of the surface displacement, velocity, and acceleration with varying order of displacement PC expansion. The results of a conventional Monte Carlo simulation - performed by deterministically propagating each of the two hundred realizations of the bedrock motion through the soil deposit for each of the five hundred realizations of the soil profile generated as described in Section 3.1 - are also shown. The stochastic finite element simulation results converge to the respective Monte Carlo result as the order of the displacement PC expansion is increased. It is also interesting to observe that a lower order PC expansion is sufficient to get a reasonable match for the surface motion for Example 2 compared to that for Example 1. This is because the marginal distributions of the surface motion in Example 2 do not deviate much from a Gaussian distribution. See, for example, Figure 10 which shows the marginal PDF of the surface acceleration time history at 8s; note that the order 1 PDF is a Gaussian distribution. These results make logical sense as in Example 2, the uncertainty in bedrock motion is much much larger than that of the shear modulus and accordingly, its characteristics can be anticipated to influence the characteristics of the surface motion much more than that of shear modulus. Recalling that the marginal distributions of the bedrock motion are Gaussian, we can reason- 
ably expect the marginal distributions of the surface motion not to deviate much from a Gaussian distribution for a linear problem like Example 2. Note that the computation time needed to solve Example 2 using the stochastic finite element method with a dimension 150, order 2 PC representation for the displacement random process - which yields a reasonable accuracy - is 3 minutes. Thus, in comparison to the computation time of about 6 hours for a hundred thousand runs of the conventional Monte Carlo method, it produces a speed-up of more than 100 .

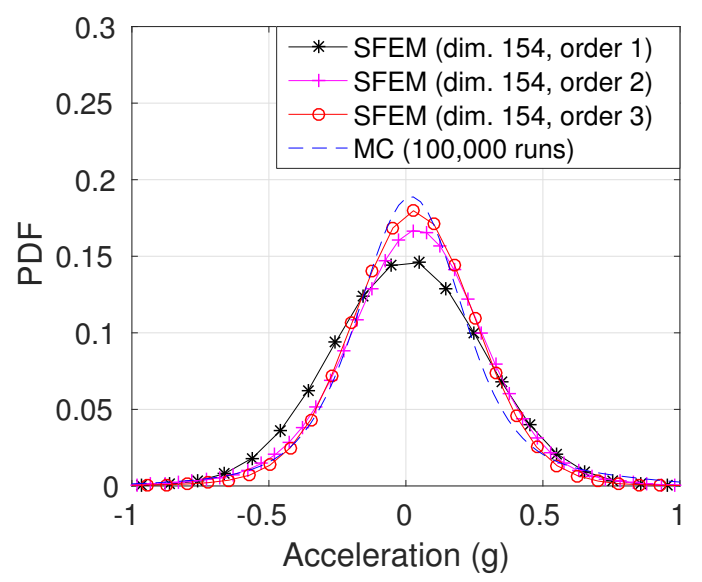

Figure 10: Simulated marginal PDF of the surface acceleration at $8 \mathrm{~s}$ when both the shear modulus and bedrock motion are uncertain.

\subsection{Comparison of Results of Example 1 and Example 2}

This subsection compares the results of Examples 1 and 2 in terms of realizations of surface acceleration time history and probabilistic characteristics of the simulated peak ground acceleration (PGA). Figure 11 shows the realization of the ground (surface) acceleration time history corresponding to the median PGA for each example. They are obtained by first generat- 
ing 100,000 realizations of the ground (surface) acceleration time history for each example from the computed surface acceleration PC coefficients, followed by sorting them according to their peak accelerations and selecting the realization corresponding to the median peak acceleration. The result of a deterministic simulation, performed by neglecting the uncertainties in soils and bedrock motion, is also shown. The deterministic simulation is carried out by assuming the soil shear modulus to be constant (100 MPa) throughout the soil deposit and using the bedrock motion shown in Figure 1. As can be observed that Examples 1 and 2 yield different median PGAs which are again different from the deterministically simulated PGA. However, from a risk analysis perspective, a more significant observation would be the spread and shape of the PDF of PGA (Figure 12). They can significantly affect the seismic risks of any structures and can only be accurately estimated by properly accounting for the uncertainties.

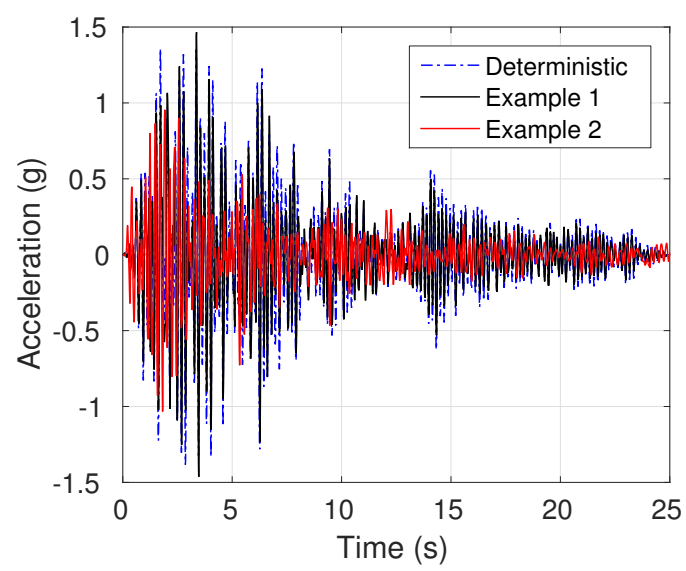

Figure 11: Simulated realization of the surface acceleration time history corresponding to the median PGA. 


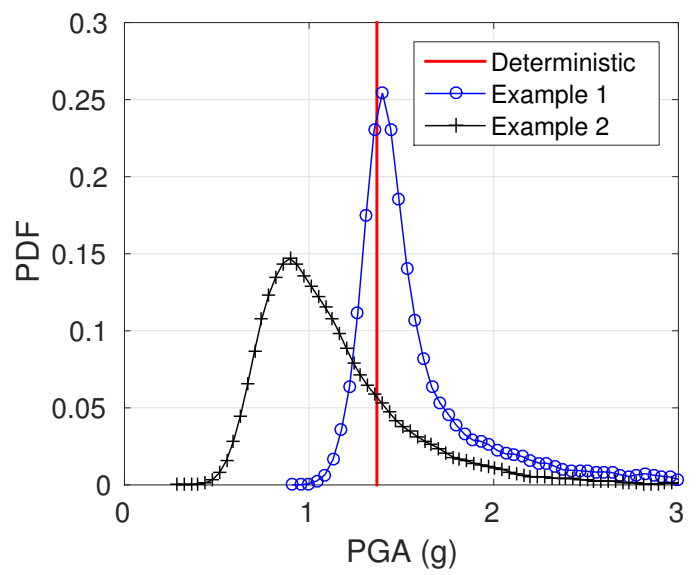

Figure 12: Simulated PDF of PGA.

\subsection{Parametric Study}

In order to quantify the sensitivity of the simulated response variables on the input uncertainty parameters, a parametric study is performed by varying the marginal coefficient of variation (COV), correlation structure, correlation length and marginal probability density function of the shear modulus random field, and nonstationary characteristics of the bedrock motion random process. Figure 13 shows the effect of the marginal COV of shear modulus random field on the simulated surface acceleration. The simulations shown in Figure 13 are performed by varying only the marginal COV of the of shear modulus random field while keeping the other input parameters the same as in Example 1. As expected, the marginal standard deviations of surface acceleration have increased, but, interestingly, its marginal mean values have decreased with increase in the marginal COV of the shear modulus. This is because the marginal PDFs of surface acceleration not only have diffused and but also have advected to the left when the marginal COV of the shear 
modulus is increased; Figure 13(c) compares the marginal PDFs of surface acceleration at $8 \mathrm{~s}$.

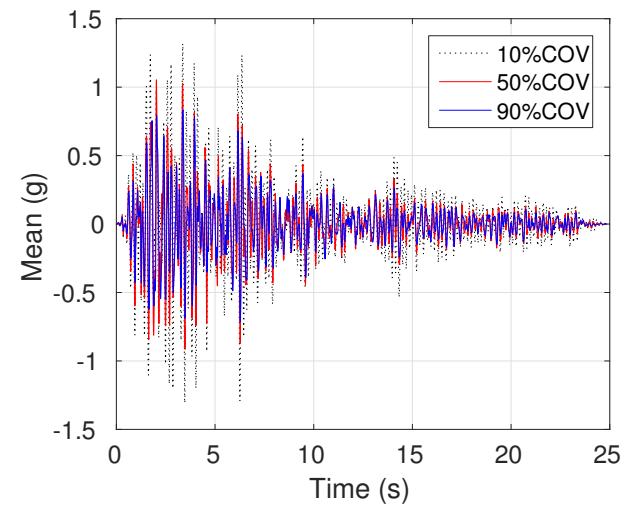

(a) Mean



(b) Standard deviation

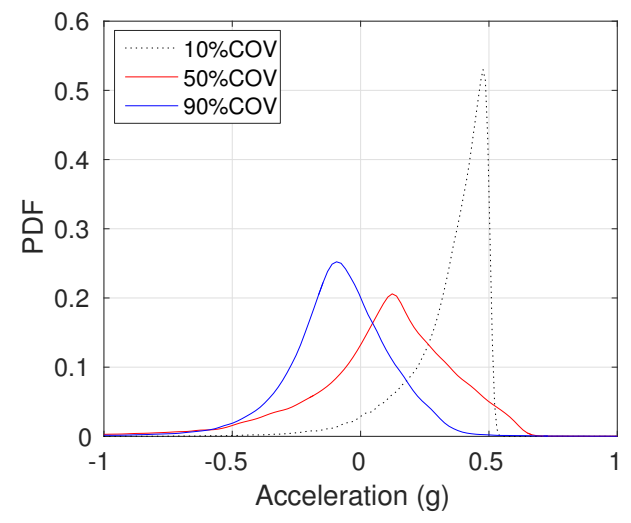

(c) PDF at $8 \mathrm{~s}$.

Figure 13: Effect of the marginal coefficient of variation of the shear modulus on simulated surface acceleration.

Figure 14 compares the simulated surface displacement for three types of shear modulus correlation structure - exponential, $\rho_{D}(x)=e^{-x / l_{c}}$ which is the same as in Example 1; triangular, $\rho_{D}(x)=1-x / l_{c}$; and exponentially damped cosine, $\rho_{D}(x)=\cos 2 x e^{-x / l_{c}}$. The correlation length $\left(l_{c}\right)$ and 
the other input parameters are kept the same as in Example 1. As can be observed, even though the choice of the correlation structure of shear modulus does not influence the marginal mean values of surface displacement, it does influence the marginal standard deviations, rather significantly for the case of exponentially damped cosine correlation structure which assumes the correlation coefficient to fluctuate from positive to negative within the same layer. Since the shape of the marginal PDF of surface displacement for the case of exponentially damped cosine correlation structure is also found to be very different, to verify, we have performed a Monte Carlo simulation too for that paricular case. As can be seen that the Monte Carlo result also shows the two-hump nature of the marginal PDF of surface displacement at 8s for the case of exponentially damped cosine correlation structure of the soil modulus.

Increasing correlation length of shear modulus random field, expectedly as random fields with larger correlation lengths yield larger variations between realizations, has resulted in increasing marginal standard deviations in simulated surface motion. The marginal standard deviation of peak surface displacement increased by approximately $90 \%$ when the shear modulus correlation length is increased from $0.5 \mathrm{~m}$ to $50 \mathrm{~m}$ (Figure 15).

The marginal PDF of shear modulus is also found to significantly influence the marginal standard deviation of surface motion (Figure 16). The peak marginal standard deviation of surface displacement for the case of uniform distribution is found to be approximately $30 \%$ larger than that for the case of lognormal distribution. It is expected as the uniform distribution assigns equal probability weights to all samples while the lognormal distribution 




(a) Mean

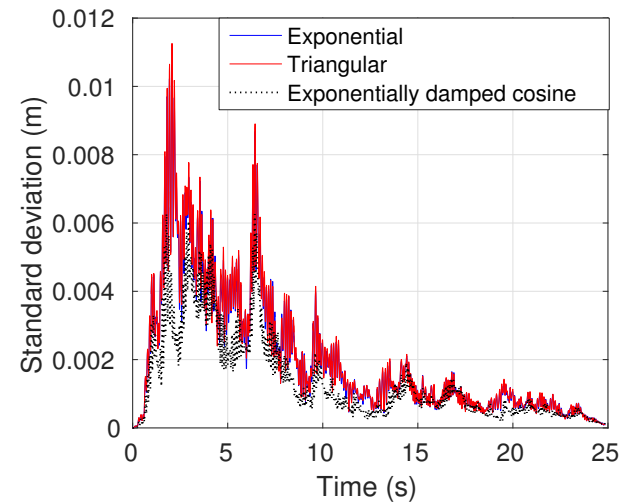

(b) Standard deviation

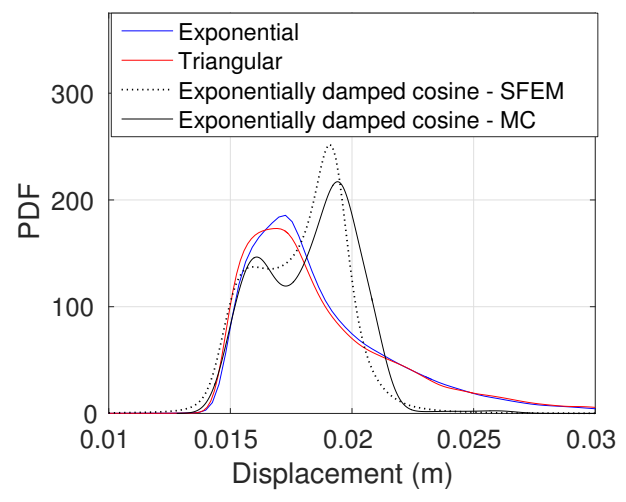

(c) PDF at $8 \mathrm{~s}$.

Figure 14: Effect of the correlation structure of the shear modulus on simulated surface displacement.

assigns very small probability weights to samples far away from the mean.

The effect of the nonstationary characteristics of bedrock random process on the simulated surface motion is also investigated. To this end, the surface acceleration simulated in Example 2 using the suite of 200 scaled, past-recorded bedrock motions is compared with that simulated using two more suites of bedrock motions obtained by spectrally matching the same 


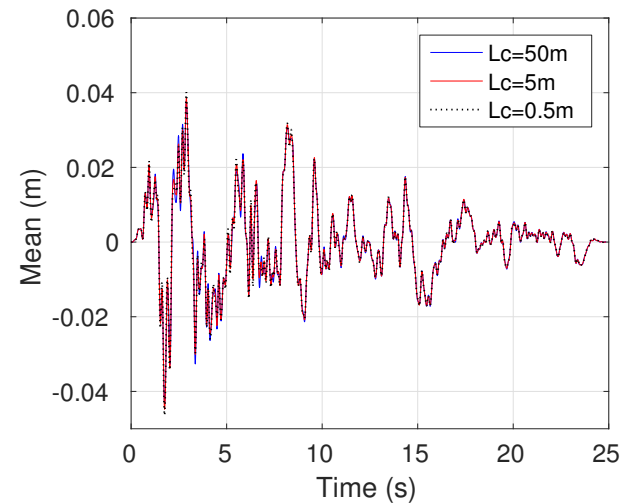

(a) Mean

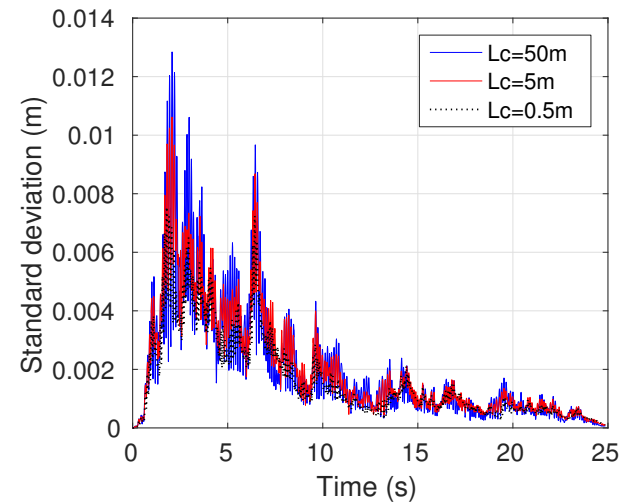

(b) Standard deviation

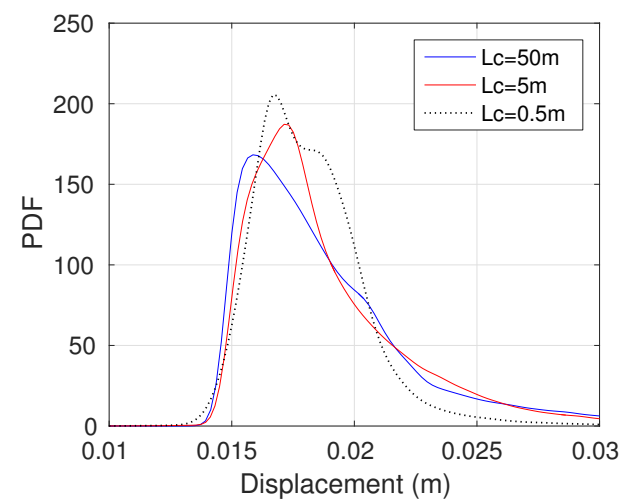

(c) PDF at $8 \mathrm{~s}$.

Figure 15: Effect of the correlation length of the shear modulus on simulated surface displacement.

site-specific response spectrum (Figure 17). One of the new suites consists of another 200 different real (scaled, past-recorded) motions while the other consists of 200 synthetic motions generated using SIMQKE [20]. As can be observed that although all three bedrock processes are generated from the same spectrum, due to their different non-stationary characteristics, each process has yielded significantly different marginal mean and marginal stan- 


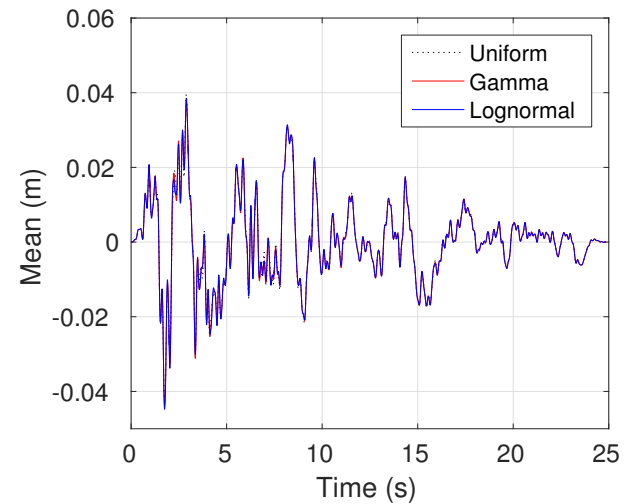

(a) Mean

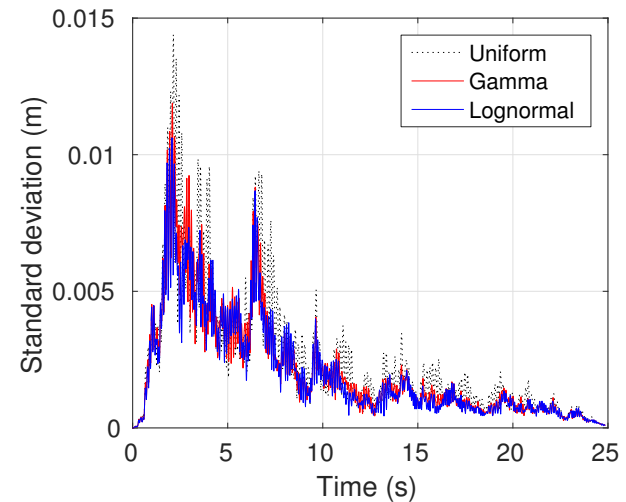

(b) Standard deviation

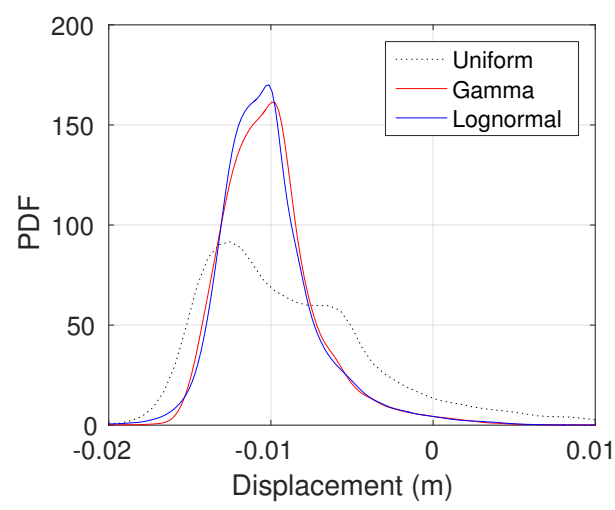

(c) PDF at $8 \mathrm{~s}$.

Figure 16: Effect of the marginal probability density function of the shear modulus on simulated surface displacement.

dard deviation behaviors of the surface acceleration.

\section{Conclusions}

An efficient finite element framework is developed for time-domain solution of the governing dynamic equations of motion in solid mechanics with the elastic modulus of the solid modeled as a heterogeneous random field 


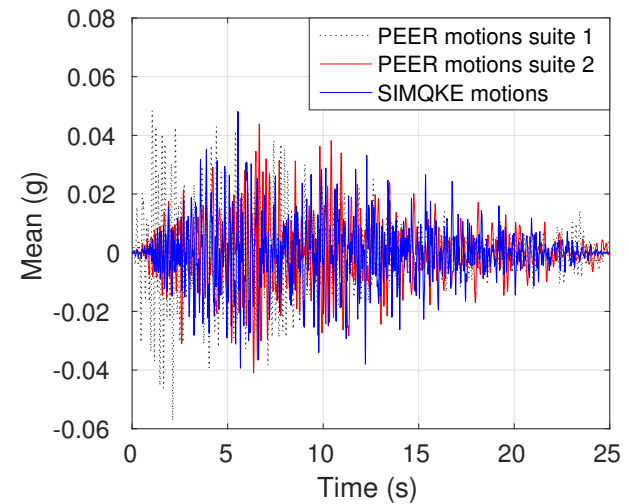

(a) Mean

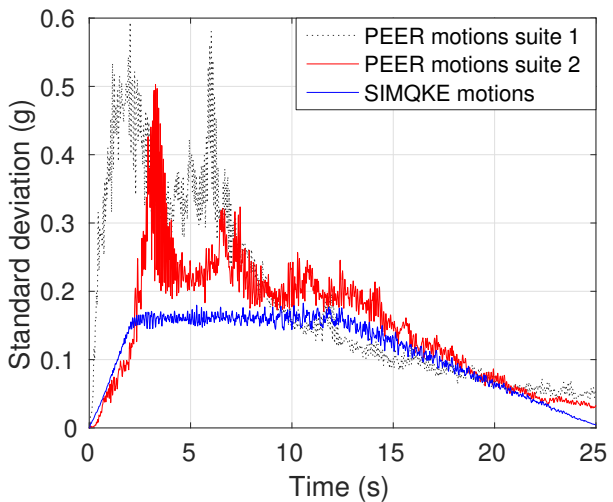

(b) Standard deviation

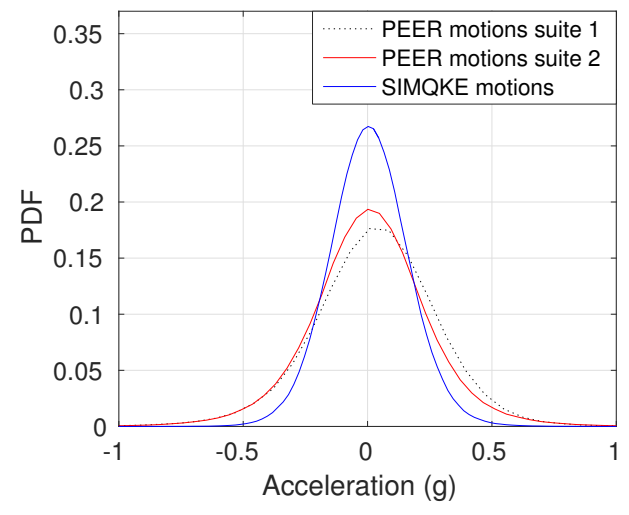

(c) PDF at $8 \mathrm{~s}$.

Figure 17: Effect of nonstationary characteristics of bedrock motion on simulated surface acceleration.

and the forcing function modeled as a non-stationary random process. The framework, which is based on an intrusive approach, first discretizes the input random process and field using multidimensional, Hermite polynomial chaos expansions. The discretizations utilize the Kosambi-Karhunen-Loève theorem for efficient representations of the covariance structures of the random process and field with optimal number of independent random variables. The 
dimensions and orders of the polynomial chaos expansions may be decided by comparing the synthesized correlation structures and marginal distributions of the input process and field with the respective target. The framework then represents the output seismic wave also in terms of a multidimensional, Hermite polynomial chaos expansion and employs a stochastic Galerkin approach in conjunction with the Newmark's time integration scheme to estimate its time-varying coefficients. The dimension of the output polynomial chaos may be chosen as equal to the total number of independent random variables used to represent the input field and process. The final form the resulting stochastic finite element equation is very similar to that of the deterministic finite element equation with the exception of the additional "stochastic" degrees of freedom arising out of the non-zeroth terms of the polynomial chaos expansion of the output seismic wave. An accuracy analysis of the Newmark's time integration scheme with the additional "stochastic" degrees of freedom has not been performed. However, a time step size typically recommended for the deterministic finite element method of about $\frac{1}{20} T_{C O}$, where $T_{C O}=2 \pi / \omega_{C O}, \omega_{C O}$ being the cut-off frequency, has been found to yield a reasonable match with the Monte Carlo result; the result of the presented stochastic finite element method converges when the order of the output polynomial chaos expansion is increased. For the examples presented in this paper, the developed framework yields speed-ups of more than 100 over the conventional Monte Carlo algorithm. Moreover, the framework is general enough to encompass any input uncertainty parameters including any non-Gaussian marginal distributions and any arbitrary correlation structures for the input seismic wave and material modulus. A parametric study, 
performed by varying the marginal COV, marginal distribution, correlation structure and correlation length of the input material modulus and nonstationary characteristics of the input seismic wave, shows that the choice of input uncertainty parameters can have significant risk implications; it not only influences the marginal standard deviations and shapes of the marginal PDFs, but also in some cases, the marginal mean values of the response variables. In closing, the time-domain formulation, as presented in this paper, seems robust and allows for, without much modifications, extension of the algorithm in solving more realistic three-dimensional as well as elastic-plastic problems.

\section{Acknowledgment}

The work presented in this paper was supported in part by a grant from the Division of Civil, Mechanical and Manufacturing Innovation of the Directorate for Engineering of the National Science Foundation (award \# NSFCMMI-1417849; cognizant program director: Dr. Richard Fragaszy).

\section{References}

[1] Acharjee, S., And Zabaras, N. A non-intrusive stochastic Galerkin approach for modeling uncertainty propagation in deformation processes. Computers $\&$ Structures 195, 5-6 (March 2007), 244-254.

[2] Anders, M., AND HorI, M. Three-dimensional stochastic finite element method for elasto-plastic bodies. International Journal for $\mathrm{Nu}$ merical Methods in Engineering 51, 4 (June 2001), 449-478. 
[3] Arnst, M., And Ghanem, R. A variational-inequality approach to stochastic boundary value problems with inequality constraints and its application to contact and elastoplasticity. International Journal for Numerical Methods in Engineering 89 (2012), 1665-1690.

[4] Babuška, I. M., Nobile, F., And Tempone, R. A stochastic collocation method for elliptic partial differential equations with random input data. SIAM Journal on Numerical Analysis 43, 3 (2007), 10051034 .

[5] Baecher, G. B., And Christian, J. T. Reliability and Statistics in Geotechnical Engineering, second ed. Wiley, West Sussex PO19 8SQ, England, 2003.

[6] Beck, J., Nobile, F., Tamellini, L., and Tempone, R. Stochastic spectral Galerkin and collocation methods for PDEs with random coefficients: A numerical comparison. In Spectral and High Order Methods for Partial Differential Equations, Lecture Notes in Computational Science and Engineering. Springer, Berlin, 2011, pp. 43-62.

[7] Beck, J., Tempone, R., Nobile, F., and Tamellini, L. On the optimal polynomial approximation of stochastic PDEs by Galerkin and collocation methods. Mathematical Models and Methods in Applied Sciences 22, 9 (September 2012).

[8] Boore, D. M. Simulation of ground motion using the stochastic method. Pure and Applied Geophysics 160 (2003), 635-676. 
[9] Bourret, R. C. Propagation of randomly perturbed fields. Canadian Journal of Physics 40 (1962), 782-790.

[10] California Department of Transportation (Caltrans). Caltrans ARS Online (v2.2.06). http://dap3.dot.ca.gov/ARS_Online/. (Retrieved May 10, 2013).

[11] Connell, C. A. Engineering seismic risk analysis. Bulletin of the Seismological Society of America 58, 5 (1968), 1583-1606.

[12] Cornell, C. A., And Krawinkler, H. Progress and challenges in seismic performance assessment. PEER Center News, Spring 2000. http://peer. berkeley. edu/news/2000spring/index.html.

[13] Cui, Y., Olsen, K. B., Jordan, T. H., Lee, K., Zhou, J., Small, P., Roten, D., Ely, G., Panda, D. K., Chourasia, A., Levesque, J., Day, S. M., And Maechling, P. Scalable earthquake simulation on petascale supercomputers. In Proceedings of the 2010 international conference for high performance computing, networking, storage, and analysis, New Orleans, LA, November 13-19, 2010 (2010).

[14] Deb, M. K., Babuska, I. M., And Oden, J. T. Solution of stochastic partial differential equations using Galerkin finite element techniques. Computer Methods in Applied Mechanics and Engineering 190 (2001), 6359-6372.

[15] DeGroot, D. J., And Baecher, G. B. Estimating autocovariance of in-situ soil properties. Journal of Geotechnical Engineering 119, 1 (January 1993), 147-166. 
[16] Elman, H. C., Miller, C. W., Phipps, E. T., and Tuminaro, R. S. Assessment of collocation and Galerkin approaches to linear diffusion equations with random data. International Journal for Uncertainty Quantification 1 (2011), 19-33.

[17] Fenton, G. A. Estimation of stochastic soil models. Journal of Geotechnical and Geoenvironmental Engineering, ASCE 125, 6 (June 1999), 470-485.

[18] Fenton, G. A. Random field modeling of CPT data. Journal of Geotechnical and Geoenvironmental Engineering, ASCE 125, 6 (June 1999), 486-498.

[19] Frisch, U. Wave propagation in random media. In Probabilistic Methods in Applied Mathematics, A. Bharucha-Reid, Ed., vol. I. Academic Press, San Diego, 1968, pp. 75-198.

[20] Gasparini, D. A., and Vanmarcke, E. H. SIMQKE: A Program for Artificial Motion Generation: User's Manual and Documentation. Department of Civil Engineering, Massachusetts Institute of Technology, 1976.

[21] Ghanem, R., and Kruger, R. M. Numerical solution of spectral stochastic finite element systems. Computer Methods in Applied Mechanics and Engineering 129, 3 (1996), 289-303.

[22] Ghanem, R. G. Ingredients for a general purpose stochastic finite elements implementation. Computer Methods in Applied Mechanics and Engineering 168 (1999), 19-34. 
[23] Ghanem, R. G., And Brzakala, W. Stochastic finite element analysis of soil layers with random interface. Journal of Engineering Mechanics 122, 4 (1996), 361-369.

[24] Ghanem, R. G., And Spanos, P. D. Stochastic Finite Elements: A Spectral Approach. Springer-Verlag, 1991. (Reissued by Dover Publications, 2003).

[25] Ghiocel, D. M., And Ghanem, R. G. Stochastic finite-element analysis of seismic soil-structure interaction. Journal of Engineering Mechanics, ASCE 118, 1 (January 2002), 66-77.

[26] Ghosh, D., Avery, P., And Farhat, C. A FETI-preconditioned conjugate gradient method for large-scale stochastic finite element problems. International Journal for Numerical Methods in Engineering 80, 6-7 (2009), 914-931.

[27] Giraldi, L., Litvinenko, A., Liu, D., Matthies, H., And Nouy, A. To be or not to be intrusive? The solution of parametric and stochastic equations - the "plain vanilla" Galerkin case. SIAM Journal on Scientific Computing 36 (2014), A2720-A2744.

[28] Hopf, E. Statistical hydromechanics and functional calculus. Journal of Rational Mechanics and Analysis 1 (June 1952), 87-123.

[29] Huang, S. P., Quek, S. T., And Phoon, K. K. Convergence study of the truncated Karhunen-Loève expansion for simulation of stochastic processes. International Journal for Numerical Methods in Engineering 52 (2001), 1029-1043. 
[30] Hughes, T. J. R. The Finite Element Method: Linear Static and Dynamic Finite Element Analysis. Dover Publications, Inc., Mineola, New York, 2000.

[31] Karapiperis, K., Sett, K., Kavvas, M. L., and Jeremić, B. Fokker-Planck linearization for non-Gaussian stochastic elastoplastic finite elements. Computer Methods in Applied Mechanics and Engineering (2015). in print.

[32] Karhunen, K. Über lineare methoden in der wahrscheinlichkeitsrechnung. Ann. Acad. Sci. Fennicae. Ser. A. I. Math.-Phys., 37 (1947), $1-79$.

[33] Kolmogorov, A. N. Über die analytischen methoden in der wahrscheinlichkeitsrechnung. Math. Ann. 104 (1931), 415-458.

[34] Kosambi, D. D. Statistics in function space. Journal of Indian Mathematical Soceity 7 (1943), 76-88.

[35] Kramer, S. L. Geotechnical Earthquake Engineering. Prentice-Hall, Upper Saddle River, NJ, 1996.

[36] Kundu, A., And Adhikari, S. Transient response of structural dynamic systems with parametric uncertainty. Journal of Engineering Mechanics 40, 2 (2014), 315-331.

[37] LaCASSE, S., AND NADim, F. Uncertainties in characterizing soil properties. In Uncertainty in Geologic Environment: From Theory to Practice, Proceedings of Uncertainty '96, July 31-August 3, 1996, Madison, 
Wisconsin (1996), C. D. Shackelford and P. P. Nelson, Eds., vol. 1 of Geotechnical Special Publication No. 58, ASCE, New York, pp. 49-75.

[38] LEE, L. C. Wave propagation in a random medium: A complete set of the moment equations with different wavenumbers. Journal of Mathematical physics 15, 9 (September 1974), 1431-1435.

[39] LoÈve, M. Fonctions aléatoires du second ordre. Supplément to P. Lévy, Processus Stochastiques et Mouvement Brownien, GauthierVillars, Paris, 1948.

[40] Mackens, W., Menck, J., And Voss, H. Coupling iterative subsystem solvers. In Scientific Computing in Chemical Engineering, F. Keil, W. Mackens, H. Voss, and J. Werther, Eds. Springer, Berlin, 1999, pp. 184-191.

[41] Manolis, G. D. Stochastic soil dynamics. Soil Dynamics and Earthquake Engineering 22 (2002), 3-15. Review Paper.

[42] Manolis, G. D., And Shaw, R. P. Harmonic wave propagation through viscoelastic heterogeneous media exhibiting mild stochasticity: I. Fundamental solution. Soil Dynamic and Earthquake Engineering 15 (1996), 119-127.

[43] Mathelin, L., And Hussaini, M. Y. A stochastic collocation algorithm for uncertainty analysis. Technical Report NASA/CR-2003212153, NASA Langley Research Center, 2003.

[44] Matthies, H. G., And Keese, A. Galerkin methods for linear and nonlinear elliptic stochastic partial differential equations. Computer 
Methods in Applied Mechanics and Engineering 194, 1 (April 2005), 1295-1331.

[45] Matthies, H. G., Niekamp, R., And Steindorf, J. Algorithms for strong coupling procedures. Computer Methods in Applied Mechanics and Engineering 195 (2006), 2028-2049.

[46] Metropolis, N., and Ulam, S. The Monte Carlo method. Journal of the American Statistical Association 44, 247 (1949), 335-341.

[47] Moenle, J., And Deierlein, G. G. A framework methodology for performance-based earthquake engineering. In Proceedings of 13th World Conference on Earthquake Engineering, Vancouver, Canada, August 1-6, 2004 (CD ROM) (2004), Paper No. 679.

[48] Newmark, N. M. A method of computation for structural dynamics. Journal of Engineering Mechanics Division, ASCE 85 (1959), 67-94.

[49] Pacific Earthquake Engineering Research Center (PeeR). Strong motion database NGA-West2. http://ngawest2. berkeley. edu/. Accessed 2015-10.

[50] Phoon, K.-K., and Kulhawy, F. H. Characterization of geotechnical variability. Canadian Geotechnical Journal 36, 4 (1999), 612-624.

[51] Phoon, K.-K., And Kulhawy, F. H. Evaluation of geotechnical property variability. Canadian Geotechnical Journal 36, 4 (1999), 625639. 
[52] Rahman, M. S., and Yeh, C. H. Variability of seismic response of soils using stochastic finite element method. Soil Dynamic and Earthquake Engineering 18 (1996), 229-245.

[53] Rezaeian, S., and Der Kiureghian, A. A stochastic ground motion model with separable temporal and spectral nonstationarities. Earthquake Engineering and Structural Dynamics 37 (2008), 1565-1584.

[54] Sakamoto, S., and Ghanem, R. Polynomial chaos decomposition for the simulation of non-Gaussian nonstationary stochastic processes. Journal of Engineering Mechanics 128 (2002), 190-201.

[55] Sett, K., Jeremić, B., and Kavvas, M. L. Stochastic elasticplastic finite elements. Computer Methods in Applied Mechanics and Engineering 200, 9-12 (February 2011), 997-1007.

[56] Soize, C. The Fokker-Planck Equation for Stochastic Dynamical Systems and its explicit Steady State Solutions. World Scientific, Singapore, 1994.

[57] United States Geological Survey (USGS). 2008 Interactive Deaggregations (Beta). https://geohazards.usgs.gov/deaggint/ 2008/. (Retrieved May 10, 2013).

[58] Wiener, N. The homogeneous chaos. American Journal of Mathematics 60, 4 (1938), 897-936.

[59] Witte, O., Roth, M., And Muller, G. Ray tracing in random media. Geophysics Journal International 124 (1996), 159-169. 
[60] XIU, D. Numerical Methods for Stochastic Computations: A Spectral Method Approach. Princeton University Press, New Jersey, 2010.

[61] Xiu, D., And Hesthaven, J. S. High-order collocation methods for differential equations with random inputs. SIAM Journal on Scientific Computing 27, 3 (2005), 1118-1139.

[62] Xiu, D., And Karniadakis, G. E. Modeling uncertainty in flow simulations via generalized polynomial chaos. Journal of Computational Physics 187 (2003), 137-167.

[63] Zabaras, N., And Ganapathysubramanian, B. A scalable framework for the solution of stochastic inverse problems using a sparse grid collocation approach. Journal of Computational Physics 227 (2008), $4697-4735$.

[64] Zhang, R. R., And Lou, M. Seismic wave motion modeling with layered 3D random heterogeneous media. Probabilistic Engineering Mechanics 16 (2001), 361-369. 